\title{
A timewise kinematic method for satellite gradiometry: GOCE simulations
}

\author{
Andrea Milani \\ Dept. Mathematics, Pisa University, Largo Pontecorvo 5, 56127 Pisa, Italy \\ e-mail:milani@dm.unipi.it
}

Alessandro Rossi

ISTI-CNR, Via Moruzzi 1, 56124 Pisa, Italy

e-mail: Alessandro.Rossi@isti.cnr.it

Daniela Villani

Hyperborea S.c., Via Giuntini 13, 56023 Cascina, Italy

e-mail:d.villani@hyperborea.com

Submitted, April 13, 2005

\begin{abstract}
We have defined new algorithms for the data processing of a satellite geodesy mission with gradiometer (such as the next European mission GOCE) to extract the information on the gravity field coefficients with a realistic estimate of their accuracy. The large scale data processing can be managed by a multistage decomposition. First the spacecraft position is determined, i.e., a kinematic method is normally used. Second we use a new method to perform the necessary digital calibration of the gradiometer. Third we use a multiarc approach to separately solve for the global gravity field parameters. Fourth we use an approximate resonant decomposition, that is we partition in a new way the harmonic coefficients of the gravity field. Thus the normal system is reduced to blocks of manageable size without neglecting significant correlations. Still the normal system is badly conditioned because of the polar gaps in the spatial distribution of the data. We have shown that the principal components of the uncertainty correspond to harmonic anomalies with very small signal in the region where GOCE is flying; these uncertainties cannot be removed by any data processing method. This allows a complete simulation of the GOCE mission with affordable computer resources. We show that it is possible to solve for the harmonic coefficients up to degree $200 \div 220$ with error to signal ratio $\geq 1$, taking into account systematic measurement errors. Errors in the spacecraft orbit, as expected from state of the art satellite navigation, do not degrade the solution. Gradiometer calibration is the main problem. By including a systematic error model, we have shown that the results are sensitive to spurious gradiometer signals at frequencies close to the lower limit of the measurement band. If these spurious effects grow as the inverse of the frequency, then the actual error is larger than the formal error only by a factor $\simeq 2$, that is the results are not compromised.
\end{abstract}

Keywords: satellite geodesy, geopotential, resonances, accelerometers, GOCE

\section{Introduction}

Gravity field and steady-state Ocean Circulation Explorer (GOCE) is an European Space Agency (ESA) mission soon to be launched, with the (C) 2005 Kluwer Academic Publishers. Printed in the Netherlands. 
purpose of determining the gravity field of the Earth to unprecedented accuracy and resolution, by using the data from two main instruments. A gradiometer measures the second derivatives of the gravitational potential. A GPS receiver measures the spacecraft position by low-high satellite-to-satellite tracking (ESA, 1999).

To extract the scientifically important results from a mission like GOCE is a large data processing task. The challenge is not just the amount of computations, but to find algorithms fully exploiting the observational information without introducing instabilities and unjustified constraints. Several different methods have been proposed to decompose the problem into feasible computational steps. The full least square fit would have a huge normal matrix: we need to find a block decomposition of the normal matrix with dominant diagonal blocks. Then the normal matrix can be approximately inverted by ignoring the off-diagonal blocks. This provides the corrections to the parameters and also an incomplete estimate of the overall covariance matrix. The procedure can be iterated to account for the neglected terms.

The selection of this decomposition requires understanding of the structure of the data and of the physics of the dynamical and measurement processes. This paper discusses both aspects. We have found an algorithm, based upon a sequence of decompositions, allowing to solve for the geopotential harmonic coefficients up to a very high degree and order with modest computational resources. We have tested such an algorithm in a full scale simulation of the GOCE data processing, including a data simulation step and a correction step. Our choices are justified both by mathematical arguments, showing the correlations and approximate symmetries which need to be controlled, and by the results of the numerical tests.

\subsection{Kinematic SOlution}

The first decomposition of the problem can be obtained from the following considerations. The GPS receivers provide phase measurements which can be processed to provide the spacecraft positions. To obtain the best results in this Precision Orbit Determination (POD) stage, the processing is usually performed with a reduced dynamical method (e.g., Visser and van den IJssel, 2000): the orbit of the spacecraft is determined only over a short arc, under a dynamical model including empirical accelerations to be solved simultaneously. The empirical accelerations absorb both the non gravitational perturbations and the inaccuracies in the knowledge of the gravitational accelerations. This process has been shown to be accurate to a few $\mathrm{cm}$ in all directions in the spacecraft position over the entire mission. 
This has two important implications: first, the data from the on board accelerometers (components of the gradiometer) do not need to be used in the POD; second, the GPS data do not provide useful information on the gravity field, because the empirical acceleration mix together the gravitational signal with the non gravitational one ${ }^{1}$. It follows that the normal matrix of the GOCE solution can be approximated by a matrix with two diagonal blocks: the normal matrix of the POD and the normal matrix for all the parameters to be solved by fitting the gradiometer data, including the geopotential coefficients $g$ and the gradiometer calibrations $\ell$. Thus it is possible to solve the full problem in two steps, the POD and the kinematic solution using the GPS orbit without corrections. This first step of the problem decomposition has been adopted by all the authors. We will discuss and test the quality of the approximation done in neglecting the dependency of the gravity field solution upon the spacecraft position errors.

\subsection{TimeWise SOlution}

A second basic choice is the organization of the GOCE observations in one of three possible ways. In a spacewise solution the data points are considered by their position in space, in a reference frame rotating with the solid body of the Earth; in this way the data are a sampling of the gravity field over a geocentric sphere (more exactly a thin shell: the orbit is almost circular). This choice has the advantage that most of the gravitational signal to be determined is also organized spatially, although there are time dependent signals due to tides and other deformations. In a timewise solution the data points are considered as a discretized time series. This is a less natural way of looking at the static part of the gravitational signal: indeed, each spherical harmonic appears as a sum of signals with different frequencies (Kaula, 1966). The advantage of the timewise methods is in the treatment of the gradiometer calibrations. In a frequencywise solution the time series of the gradiometer data are Fourier transformed into the frequency domain. Since the effect of each spherical harmonic can be represented by a Fourier polynomial with good approximation, the fit can be directly performed in the frequencies space. This method is the most convenient in the mission design phase, because it allows to convert requirements in the error spectrum of the gradiometer into error spectrum of the recovered gravity coefficients.

${ }^{1}$ There are other methods of data processing to extract gravity field information from the GPS data, as used with CHAMP (Reigber et al., 2002), but they cannot be pushed to the GOCE level of accuracy and resolution. 
These three approaches are equivalent for a perfect distribution of data. With a spatial distribution uniform on a sphere and a time distribution uniform over an unlimited time span there is a well conditioned one-to-one correspondence between spherical harmonics, a linear subspace of the signal as function of time, and its discrete spectra. However, such uniformities are impossible in real satellite geodesy missions. The distinction of the spacewise approach has been well understood since the early phases of GOCE (Rummel et al., 1993), but the implications of the difference between the timewise and the frequencywise methods have been underestimated.

A fundamental problem of all the satellite geodesy missions with on board accelerometers of whatever type is that all such instruments provide relative measurements ${ }^{2}$. Thus it is necessary to add a posteriori calibration parameters to the list of parameters to be determined. The instrumental calibrations are organized timewise: the accelerometer biases can be assumed to change smoothly with time, while if they were organized spatially they would appear almost random.

The calibrations of the individual accelerometer channels can be combined into calibrations for the gradiometer and calibrations for the common mode measurements. The latter are obviously strongly correlated with the empirical accelerations solved in the reduced dynamics POD. The gradiometer calibrations can be determined neither from the orbit nor from the common mode calibrations.

Several methods have been proposed to remove the effect of the gradiometer errors, including calibration errors, from the data. In a frequencywise method digital filters can be used; however, as pointed out in Albertella et al. (2001), the frequency domains of the calibrations and of the gravitational signal have some overlap, thus the procedure has to be much more than a simple linear filtering. Moreover, the frequencywise methods are very sensitive to data gaps (in time). In a spacewise method, the calibration error can be partially removed by averaging/smoothing techniques, but there is no guarantee that systematic errors would be fully removed. It is clear that solving for the calibration error is by far simpler within a timewise method.

Our method can be described as timewise kinematic, in that the orbit is assumed (from the POD) and the predicted gradients are computed as a time series and directly compared with the individual observations. The least squares fit is linear; nevertheless it may require iterations because the normal system of equations is too large to be

\footnotetext{
${ }^{2}$ Absolute a priori calibration of an accelerometer is possible only at moderate levels of accuracy (such as in inertial guidance systems). In flight calibration is possible with dedicated devices, but to achieve the level of accuracy envisaged for the GOCE measurements would be very difficult.
} 
solved at once, as discussed in Section 4.3, but the number of necessary iterations is small.

The separation of the calibrations from the gravity signal is the second step of the problem decomposition. It is obtained by fitting the calibrations to a linear combination of slowly varying base functions, separately on each time interval: it is described in Section 2. This allows the separation of the calibrations as local parameters from the global ones describing the gravity field, as discussed in Section 3. A third decomposition step is necessary to allow the full solution to be computed with limited computing resources. With a method called resonant decomposition we reorder the normal matrix of the $g$ variables in such a way that it also has a dominant block diagonal structure: this is described in Section 4.

A timewise method is insensitive to data gaps organized by time (e.g., instrument shutdown/communication failures), but all methods are sensitive to data gaps organized spatially. We show in Section 5 that the polar gaps resulting from the non polar orbit of GOCE introduce an exact symmetry in the functional space of spherical harmonics. This exact symmetry is broken in a finite spherical harmonics expansion, but still holds as an approximate symmetry. Thus all data processing methods have to be sensitive to the indetermination resulting from the polar gaps. We validate the results of our numerical experiments in Section 6 by showing that the main indetermination is the one unavoidably resulting from the polar gaps.

\section{Gradiometer Calibration}

The observations include two independent components of the gravity gradient. E.g., if they are sampled every $10 \mathrm{~s}$ for a measurement time span of about 8 months $^{3}$, the observations form a vector $A$ with $\simeq 4 \times 10^{6}$ components. We have performed a fit to the gradiometer observations vector $A$ solving for two parameter vectors: the gravity harmonic coefficients $g$ (e.g., $201^{2}$ coefficients if the field is determined up to degree and order 200) and the gradiometer calibration parameters $\ell$. The main issue is the dimension of $\ell$. E.g., let us suppose a set of 2 calibration parameters had to be solved for each interval of $\simeq 200 \mathrm{~s}$, since $f_{m}=1 / 200 \mathrm{~Hz}$ is the lower frequency limit of the measurement band, where the gradiometer data are most accurate (ESA, 1999, Section 8.1): this would imply $\simeq 2 \times 10^{5}$ calibration parameters. This large number

\footnotetext{
${ }^{3}$ This sampling is meant to be the result of a preprocessing stage, in which digital filtering is used to remove the noise at frequencies higher than the upper limit $f_{s} \simeq 1 / 10 \mathrm{~Hz}$ of the measurement band.
} 
would be a problem, not only for the computational load (especially memory size), but also for the bad conditioning of the normal matrix. Thus we need to discuss in depth the calibration requirements.

\subsection{NeEd FOr a POSTERIORI CALIBRATION}

The measurement error of a space borne gradiometer is not dominated by noise, but by systematic signals, mostly from two sources.

Thermal signals arise from the basic physical property of accelerometer devices: they are anyway also thermometers. The GOCE accelerometer units have a thermal response of $\simeq 10^{-5} \mathrm{~cm} / \mathrm{s}^{2} / \mathrm{K}^{-1}$. If the changes in temperature had a noise behavior, the spurious signal introduced in the accelerometer readings by the thermal effects would appear as a noise, to be described in terms of power spectrum. However, thermal signals are by no means random, they occur with well defined frequencies depending upon the external sources of heat and the control cycles of the thermal stabilization system ${ }^{4}$.

The second main source of gradiometer measurement error is the attitude control system. A gradiometer cannot measure the gravity gradient independently from the gradient of the apparent forces due to the spacecraft rotation (ESA, 1999, Sect. 7.2, Bouman and Radboud, 2003). The centrifugal term can be deduced from the sum of the three diagonal terms in the gravity gradient matrix, this is the reason why only two diagonal components can be independently measured with full precision. However, there are error terms induced by the inaccuracy of the attitude control and knowledge, and by the inaccurate knowledge of the location of the accelerometers with respect to the spacecraft center of mass. The attitude control system operates with a characteristic, highly colored, frequency spectrum.

Thus both the main sources of error introduce a very systematic time dependent bias. One important component of the gradiometer error cannot be modeled as a noise with a continuum spectrum, but rather as a superposition of oscillations with well defined periods, such as the orbital period and its submultiples, the day, the year and its submultiples (Pail and Plank, 2002). From this we draw two conclusions. First, a model for these systematic biases needs to be included in the data simulation, otherwise the results would show an illusory accuracy. Second, this bias, at least for the low frequency components, needs to be digitally removed by solving for calibration parameters in the correction step.

\footnotetext{
${ }^{4}$ Thermal stabilization systems cannot control the absolute temperature, they cannot avoid long term drifts in the temperature, because an absolute thermometer is as challenging as an absolute accelerometer.
} 


\subsection{Simulation of Systematic CAlibration ERrors}

Although the measurement errors of the gradiometer contain also noise, we are mostly interested in evaluating the effects of the systematic bias components $^{5}$. Thus we have used, in the data simulations, an error model with a systematic component as well as a random one. For the random component we have used uncorrelated Gaussian noise with RMS $0.004 \mathrm{E}$ (Eötvös units, $10^{-9} \mathrm{~s}^{-2}$ ). This noise term is used to define the formal covariance, that is the gravity gradient residuals are weighed dividing by $0.004 \mathrm{E}$.

The systematic component has been modeled by a finite number of harmonics, with an amplitude inversely proportional to the frequency, up to a period of one day. This includes a daily term (with amplitude of $1.73 \mathrm{E}$ ), a once per rev (with amplitude of $0.1 \mathrm{E}$ ) and a twice per rev terms (with amplitude of $0.055 \mathrm{E}$ ), supposedly accounting for thermal changes. For the very long term drift (supposedly due to seasonal thermal effects) we have assumed a period of one year and an amplitude of $18.6 \mathrm{E}$, decreased, with respect to the $f^{-1}$ law, by a factor 0.03 (supposedly an a priori calibration by means of a temperature measurement accurate to $\simeq 2$ milli Kelvin). We have also added a term with period $1,000 \mathrm{~s}$ and amplitude of $0.02 \mathrm{E}, 5$ times the RMS of the noise component, with the purpose of investigating the systematic errors introduced in the solution for the harmonic coefficients as a result of spurious signals due to other causes, such as the attitude control.

\subsection{Fit of CALibration PARAMETERS}

The time dependent gradiometer bias, for each of the two independent components, can be represented as a linear combination

$$
b(t)=\sum_{i=1}^{N} d_{i} c_{i}(t)
$$

of suitable base functions $c_{i}$ of the time. This representation is applicable only to a limited time span, which we will call an arc. The need to decompose the fit into arcs arises from two considerations. First, the number $N$ of base functions solved at once cannot be too large to avoid computational complexity and instability. Second, we do not want to impose one specific functional representation of the bias over the entire mission duration, because real information on the long term behavior of the accelerometer is not available. Of course in the data simulation

\footnotetext{
${ }^{5}$ This also because the effects of noise have already been discussed by other authors, e.g., (Pail and Plank, 2002)
} 
we introduce biases with a specific functional form, e.g., a finite sum of harmonics as discussed in the previous subsection, but this information must not be used in the correction step.

On the other hand the time span $\Delta t$ over which the representation of Eq. 1 is applicable cannot be too short. The bias to be removed in this way has to be the low frequency component, with frequencies below a calibration band upper limit $f_{c}$ well separated from the measurement band lower limit $f_{m} \simeq 1 / 200 \mathrm{~Hz}$. As an example, the tests of this paper have used a calibration band with frequencies below $f_{c}=1 / 2000$ $\mathrm{Hz}$. Thus the combination of values of $\Delta t$ and $N$, and the set of base functions $c_{i}$, have to be selected to model a bias with arbitrary signal in the calibration band. Note that the spurious signals in the intermediate band with frequency between $f_{c}$ and $f_{m}$ are not removed.

The choice for the base functions needs to take into account three requirements. First, the total number of parameters needs to be controlled, with an appropriate choice of the parameters $\Delta t$ and $N$. Second, the normal system for all the calibration parameters $\ell$ needs to be well conditioned. Third, we need to stress once again that the calibration model should not constrain the shape of the calibration as a function of time, and should not use information on this shape illegitimately transfered from the simulation step.

A natural choice would be to use a Fourier polynomial representation of the calibrations over the arc time span, with a combination of a constant and sine and cosine terms with periods $\Delta t / k$ for $k=1, \ldots, K$; then $N=2 K+1$. To limit the removal of signal to the calibration band, $K=\Delta t \cdot f_{c}$. E.g., $\Delta t=10,000 \mathrm{~s}, K=5$ are acceptable choices. The second requirement is met because the Fourier terms are orthogonal:

$$
\int_{t_{0}}^{t_{0}+\Delta t} \cos (k t) \cdot \cos (j t) d t=0
$$

for $k \neq j$, and similarly for the sine terms. The normal matrix of the coefficients $c_{i}, i=1, \ldots, 2 K+1$ contains finite sums approximating the above integrals, thus it is diagonal dominant. However, this selection of base functions does not satisfy the third requirement, because all the base function are periodic of period $\Delta t$ and thus have the same value at the two extremes of the arc interval. If a calibration with a long term trend was fitted in this way, the post fit residuals would show comparatively large "jumps" at the arc boundaries. To mitigate this effect we have added a linear function of time to the base functions. Thus the number of coefficients per arc is increased to $2 K+2$, the normal matrix is not anymore diagonal dominant but the conditioning remains acceptable, and the "jumps" are significantly reduced, although they are still present. 
It would be possible to reduce the arc boundary "jumps" to levels below the noise, by fitting a calibration model with margins beyond the arc boundaries, that is with Fourier components of periods $\Delta t \cdot(1+$ $2 h) / k$ over the $\operatorname{arc} t_{0}-h \Delta t \leq t \leq t_{0}+(1+h) \Delta t$. Then the calibration model would be used only on $t_{0} \leq t \leq t_{0}+\Delta t$, removing the spurious boundary effects. This strategy for digital calibration would produce residuals of the final fit without the few comparatively high values due to the jumps, but no substantial changes would result on the solution for the gravity coefficients (see Section 6.1).

Another possibility would be to use Chebychev polynomials as base functions, and indeed this would result in much lower "boundary jumps", but we have found that the results would be somewhat worse in terms of solution for the gravity coefficients.

In conclusion, the best strategy to fit the calibration would be to use Fourier polynomials, augmented with a linear function, with margins beyond the arc boundaries. However this would make the multi arc solution, discussed in Section 3.1, more complicated. For this reason we have performed most simulations without margins beyond the arc boundaries, but we have tested the sensitivity of the results to this problem (see Section 6.1).

The total number of calibration parameters $\ell$ for a simulation over a total time span of $2 \times 10^{7} \mathrm{~s}$ is therefore

$$
2 \times(2 K+2) \times N_{\text {arc }}=2 \times 12 \times 2 \times 10^{7} / 10^{4}=48,000
$$

where $N_{\text {arc }}$ is the number of arcs.

\section{Multi arc solution}

The multi arc approach is a form of the least squares method, in which the list of parameters is split in accordance to the partition of the orbit into arcs (Reigber, 1989; Milani and Melchioni, 1989; Milani et al., 1995). We briefly recall the necessary formulae in the next subsection.

\subsection{LOCAL-GLOBAL DECOMPOSITION}

We use the notation $[a ; b]$ to indicate the stacking of the two column vectors $a$ and $b$ to form a longer vector. The vector of all parameters $z=[g ; \ell]$ is split into a vector $g$ of global solve-for parameters, in this case the harmonic coefficients of the gravity field, and a vector $\ell$ of local solve-for parameters, in this case the gradiometer calibration parameters. $\ell$ is further split into vectors $\ell_{j}$, one for each arc, formed by the gravity gradient measurements over a given span of time; also 
the residuals $\xi$ are split in the $\xi_{j}$, where $j=1, \ldots, N$ is the arc index. The residuals from one arc do not depend upon the local parameters of another arc:

$$
B_{g}^{(j)}=\frac{\partial \xi_{j}}{\partial g} \quad ; \quad B_{\ell_{i}}^{(j)}=\frac{\partial \xi_{j}}{\partial \ell_{i}}=\underline{0} \quad \text { for } i \neq j
$$

where the residuals are already normalized. As a result the contributions of each arc to the overall normal system are as follows:

$$
\begin{aligned}
C_{\ell_{i} \ell_{j}} & =\left(B_{\ell_{j}}^{(j)}\right)^{T} B_{\ell_{i}}^{(i)}=C_{\ell_{j} \ell_{i}}^{T} \quad \neq \underline{0} \text { only for } i=j \\
C_{g \ell_{i}} & =\left(B_{g}^{(i)}\right)^{T} B_{\ell_{i}}^{(i)}=C_{\ell_{i} g}^{T} \quad ; \quad C_{g g}=\sum_{i=1}^{N}\left(B_{g}^{(i)}\right)^{T} B_{g}^{(i)}=C_{g g}^{T}
\end{aligned}
$$

giving to the normal system matrix $C$ an arrow-like structure (Colombo, 1989). The right hand side of the normal system can be computed by

$$
D_{g}=-\sum_{i=1}^{N}\left(B_{g}^{(i)}\right)^{T} \xi_{i} \quad ; \quad D_{\ell}=\left[D_{\ell_{1}} ; \ldots ; D_{\ell_{N}}\right] \quad ; \quad D_{\ell_{i}}=-\left(B_{\ell_{i}}^{(i)}\right)^{T} \xi_{i}
$$

Then the normal system can be written as a system of two equations:

$$
\left\{\begin{array}{l}
C_{g g} \Delta g+C_{g \ell} \Delta \ell=D_{g} \\
C_{\ell g} \Delta g+C_{\ell \ell} \Delta \ell=D_{\ell}
\end{array}\right.
$$

To solve exactly the normal system above without losing the advantage of the separation between local and global parameters, we solve the second equation with respect to the differential correction $\Delta \ell$ of the local parameters, then substitute into the first one:

$$
\left\{\begin{array}{l}
\Delta \ell=C_{\ell \ell}^{-1}\left[D_{\ell}-C_{\ell g} \Delta g\right] \\
{\left[C_{g g}-C_{g \ell} C_{\ell \ell}^{-1} C_{\ell g}\right] \Delta g=D_{g}-C_{g \ell} C_{\ell \ell}^{-1} D_{\ell}}
\end{array}\right.
$$

As a result, the solution for the differential correction $\Delta g$ of the global parameters is obtained by means of the variance-covariance matrix $\Gamma_{g g}$ :

$$
\Gamma_{g g}=\left[C_{g g}-C_{g \ell} C_{\ell \ell}^{-1} C_{\ell g}\right]^{-1} ; \Delta g=\Gamma_{g g}\left[D_{g}-C_{g \ell} C_{\ell \ell}^{-1} D_{\ell}\right] .
$$

The corrections $\Delta g$ and the covariance $\Gamma_{g g}$ are not the same as it would be obtained from a separate global-only correction (that is, $\Gamma_{g g} \neq C_{g g}^{-1}$ ). This because of the correlation between the local and the global parameters, which is not zero and can be computed by:

$$
\Gamma_{\ell_{i} g}=-C_{\ell_{i} \ell_{i}}^{-1} C_{\ell_{i} g} \Gamma_{g g} .
$$

The corrections $\Delta \ell$ are found by the first of the two normal equations: they are not the same as in a local-only correction. 
If the $\ell$ parameters satisfy Eq. (3), then the matrix $C_{\ell \ell}$ is block diagonal and can be inverted block by block. If there are some residuals from one arc depending upon the calibration parameters of a neighboring arc, as it is indeed the case when the calibration model is fitted with margins beyond the arc boundaries, then the matrix $C_{\ell \ell}$ is block tridiagonal and the procedure to invert it becomes more demanding.

\subsection{RelevanCE of THE LOCAL-GLOBAL CORRELATION}

It would be convenient to split the solution into two independent steps, first to determine the calibration parameters $\ell$, then to solve for the gravity parameters $g$. This, however, ignores the portion $C_{\ell g}$ of the normal matrix, forcing $\Gamma_{\ell g}=0$, and changes the solution $\Delta g$.

The replacement of $\Gamma_{g g}$ with $C_{g g}^{-1}$ is the most dangerous approximation, because it is potentially misleading. Eq. (4) shows that the true $\Gamma_{g g}^{-1}$ is $C_{g g}$ minus a symmetric positive definite matrix. Geometrically, the confidence ellipsoid surrounding the nominal solution $g^{*}$ is

$$
Z(\chi)=\left\{g \mid\left(g-g^{*}\right)^{T} \Gamma_{g g}^{-1}\left(g-g^{*}\right)<\chi^{2}\right\}
$$

for a given confidence level $\chi$. If $C_{g g}^{-1}$ was used instead of $\Gamma_{g g}$, then the confidence ellipsoid would be believed to be the ellipsoid defined by the matrix $C_{g g}$ instead of $\Gamma_{g g}^{-1}$, which is a proper subset of the ellipsoid $Z(\chi)$. Then the solution would be deemed to belong to the smaller ellipsoid, while it actually belongs to the larger one. To avoid results with a claimed illusory precision, we need to either perform the computation at once for $\ell$ and $g$, or to test the difference between the full computation and the simplified one and show it is irrelevant. The experience with other satellite geodesy missions has shown that to forget this check could be disastrous (Milani et al., 2001).

The results of one such test is shown in Figure 1. We have computed a full solution, including local-global correlations, and we plot in the Figure, as a function of the degree $l$, both the formal variance and the actual error (that is the difference between the solution of the differential corrections and the "true" value used in the data simulation). Then we have computed a solution by zeroing the sub-matrix $C_{\ell g}$, equivalent to two separate solutions for $\ell$ and for $g$ : the formal variance is significantly smaller than the one from the complete computation, especially for $l=2,3,6,7,8$. The difference becomes not significant for $l \geq 25$. The local-global correlations (from the matrix $\Gamma_{\ell g}$ ) are $\simeq 0.2$ for the Fourier component of the bias with $k=4$, that is with frequency $1 / 2500 \mathrm{~Hz}$ and the $l=2, m=0$ harmonic. As a result of the correlations, the RMS uncertainty of the $(2,0)$ harmonic is increased by 


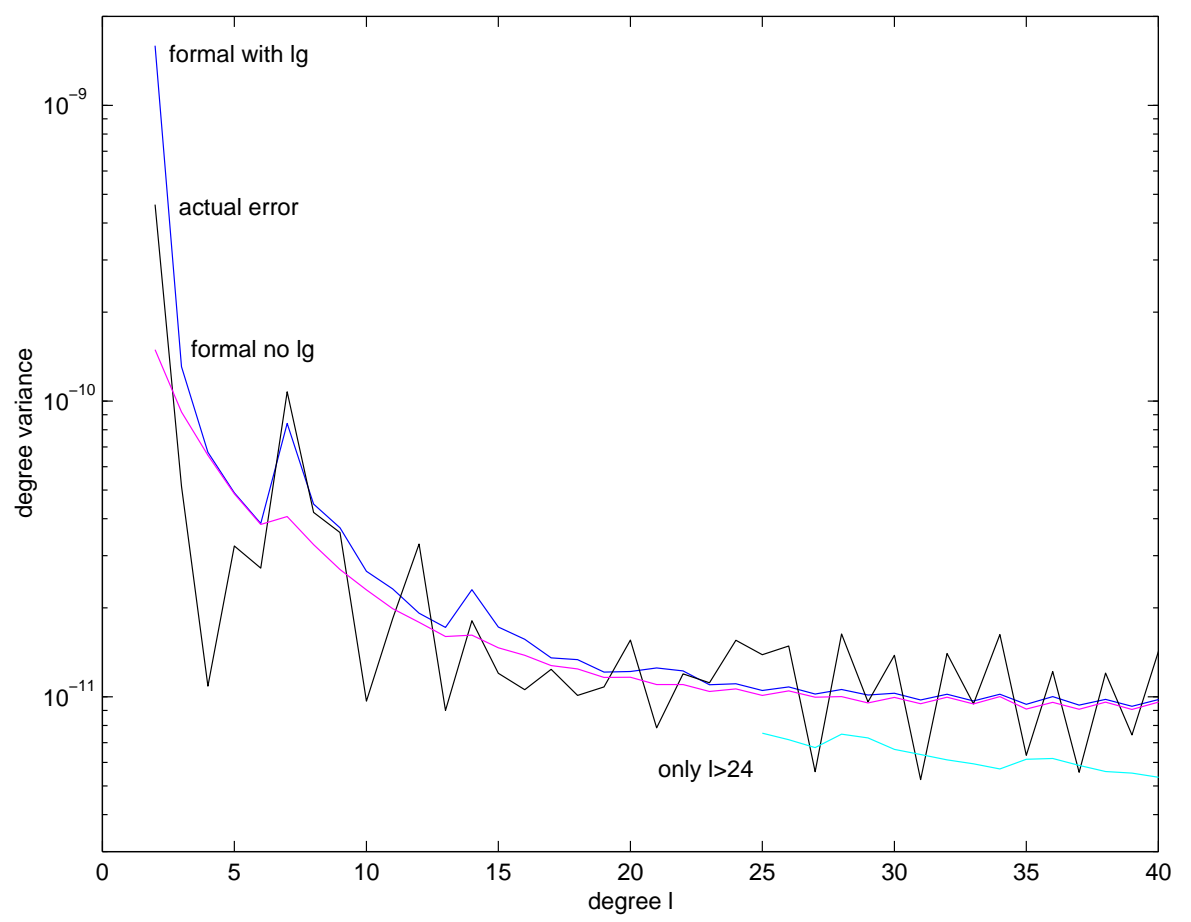

Figure 1. Comparison of different results for the spherical harmonic gravity coefficients, as a function of the degree $l$. Starting from the top at the left end of the plot: the formal RMS from the covariance matrix computed including the local-global correlations; the actual error of the computed solution; the formal RMS from the covariance matrix computed without the local-global correlations. The formal RMS of the computation with local-global limited to $l>24$ appears at the lower right.

an order of magnitude. The actual error is consistent with the higher formal uncertainty, statistically incompatible with the lower estimate.

The conclusion of this test is that the local-global correlations are not negligible at all, at least for the low degrees. These results have also an interpretation in terms of signal frequencies (see Figure 4 in Albertella et al., 2001). The spherical harmonics up to degree $\simeq 25$ generate gradiometer signals with frequencies lower than those of the measurement band. In particular, the harmonics of degree 2 generate signals in the calibration band (frequencies lower than $f_{c}=1 / 2000 \mathrm{~Hz}$ ) which can be absorbed in the estimated calibrations.

Thus GOCE should not be used to solve for the gravity coefficients of low degrees. Several methods can be used to avoid the problems resulting from the local-global correlation. The simplest method is to solve only for coefficients of degree $l>24$ : then the effect of the local- 
global correlations is not significant (negligible in the variance, less than $10 \%$ in the actual error). The result of this $l>24$ solution is the curve in the lowest right corner of Figure 1: the variance is somewhat underestimated, due to the correlations between the harmonics with $l>24$ and the ones with $2 \leq l \leq 24$; this effect becomes negligible for harmonics with $l>75$. Thus, for the purpose of these simulations, with the main goal of computing a reliable estimate of the error for high degrees, this approximation is sufficient.

In the processing of the real data it would be better to use the collocation method, e.g., by adding a priori observations of the harmonics with $2 \leq l \leq 24$ weighed with the inverse of their known covariance matrix, as resulting from the analysis of other missions. The analysis of the CHAMP and GRACE data should provide more than enough a priori information before the launch of GOCE.

We can conclude that, if the arc length $\Delta t$, the number of calibration parameters per arc $2 K+2$, and the minimum degree $l$ are chosen properly, then it is possible to solve separately for the calibration parameters $\ell$ and then to solve for the harmonic coefficients $g$ only.

\section{Resonant Decomposition}

As a result of the discussion in the previous Section, it is always possible to compute the covariance matrix of the scientifically interesting results, that is $\Gamma_{g g}$, in a separate step, either simply as $C_{g g}^{-1}$ or by using Eq. (4). If the goal is to determine coefficients up to a large degree, e.g., $l=200$ or even $l=250$, the matrix to be inverted is still very large.

This results into two types of problems. First, practical problems of computational resources. It is cumbersome to invert a matrix which cannot fit in the RAM of the computer, and to store a matrix such as $C_{g g}$ for degree up to $l=250$ requires more than $15 \mathrm{G}$ Bytes. Also the computing time for such a global solution (with a single CPU) would introduce problems of reliability. This makes difficult to perform this computation without using very expensive hardware. Second, problems of numerical stability. The larger the matrix, the higher the risk that the conditioning number is horrendous, and the results, even with a state of the art supercomputer, would be degraded. For reasons discussed in the next Section, we do know that $C_{g g}$ is badly conditioned.

Thus it is necessary to decompose the problem into smaller ones: we need to approximate the solution of the normal system

$$
\Gamma_{g g}^{-1} \Delta g=D_{g}
$$




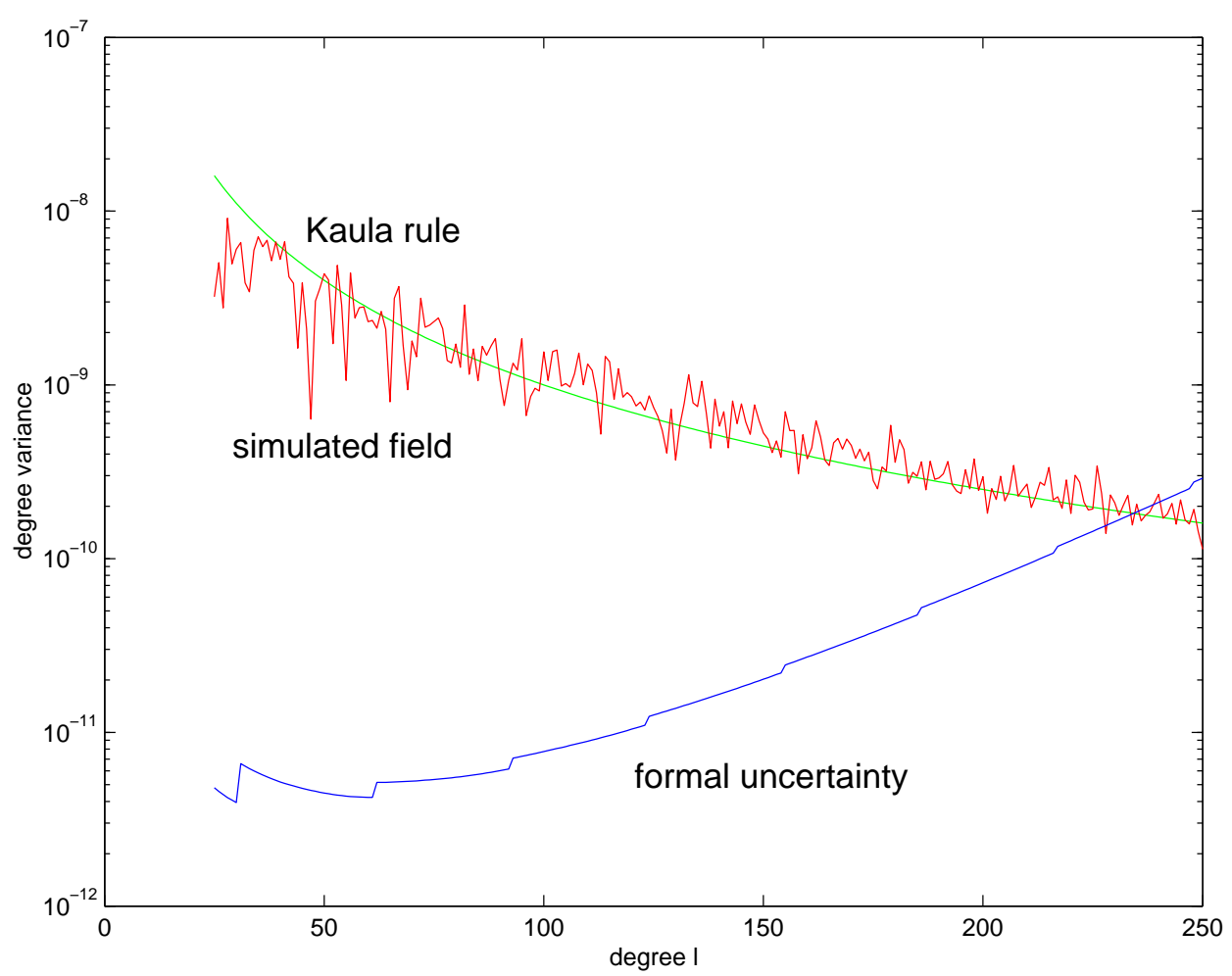

Figure 2. Formal standard deviation of the spherical harmonic gravity coefficients as a function of the degree $l$, for $25 \leq l \leq 250$, including in the estimate only the diagonal terms of the normal matrix $C_{g g}$. The coefficients included in this test are only the ones for the remainder class $r=0$ (see Section 4), including the zonals.

with a sequence of independent differential corrections for suitably chosen subsets of the $g$ parameters.

A trivial decomposition would be to solve for the harmonic coefficients one by one, that is, to approximate the $C_{g g}$ normal matrix with its main diagonal. Since the spherical harmonics are orthogonal on a sphere and the GOCE measurements are roughly at constant altitude, it might be expected that the terms outside the main diagonal are small. However, this would be true if the GOCE measurements were taken over a complete sphere (and with uniform spatial density). As a result of the uneven spatial distribution of the measurements some off diagonal terms are as large as the diagonal ones, the matrix is not at all diagonal dominant, and the computation of the coefficients one by one is a very poor approximation.

In Figure 2 we show the result of such a simplified computation, compared with the signal assumed in the simulation (taken from the EGM96 geopotential model, Lemoine et al., 1998) and Kaula's rule. The 
curve show the formal RMS in the diagonal approximation, that is the inverse square root of the diagonal term in $C_{g g}$, for a subset of gravity coefficients including the zonal $(m=0)$ harmonics for $25 \leq l \leq 250$. A comparable plot showing the results of a full computation is Figure 4, and it is clear that the two formal estimates are different by two orders of magnitude for intermediate degrees. We conclude that to solve the coefficients one by one would lead to claims of illusory precision.

Thus it is necessary to identify the subsets of the harmonic coefficients which are significantly correlated. If the vector $g$ is reordered by using these correlated subsets, an almost block diagonal structure appears, that is, the elements of the matrix outside the diagonal blocks are not zero but much smaller than the corresponding elements in the blocks. Then the matrix with the diagonal blocks and zeros outside the blocks is a good approximation of $C_{g g}$. This Section presents one very effective method to find this correlated subset decomposition, based on (Milani et al., 1998).

\subsection{FREQUENCY ANALYSIS OF THE SPHERICAL HARMONICS}

The Earth gravity potential $U$ can be developed in the spherical harmonic terms $U_{l m}$ of degree $l(l \geq 0)$ and order $m(m=0, \ldots, l$; "real representation"). When transformed in the orbital elements of the satellite the contribution of a particular harmonic $(l, m)$ reads (Kaula, 1966)

$$
U_{l m}=\frac{G M_{e}}{R_{\oplus}} \sum_{p=0}^{l} \sum_{q=-\infty}^{\infty}\left(\frac{R_{\oplus}}{a}\right)^{l+1} \bar{F}_{l m p}(I) G_{l p q}(e) A_{l m} \cos \left(v_{l m p q}-\psi_{l m}\right)
$$

where $M_{e}$ is the Earth mass, $R_{\oplus}$ its radius, $a$ the semimajor axis of the satellite orbit, $e$ the eccentricity and $I$ the inclination. $\bar{F}$ and $G$ are the normalized inclination and eccentricity functions. The amplitudes $A_{l m}$ and phases $\psi_{l m}$ are obtained from the normalized geopotential coefficients $\bar{C}_{l m}$ and $\bar{S}_{l m}$ (the global parameters $g$ ) by

$$
A_{l m} \exp \left(i \psi_{l m}\right)=\bar{C}_{l m}+i \bar{S}_{l m},
$$

and the argument $v_{l m p q}$ is given by

$$
v_{l m p q}=(l-2 p) \omega+(l-2 p+q) M+m\left(\Omega-\vartheta_{e}\right)-\frac{\pi}{2} \bmod (l+m, 2)
$$

where $(\Omega, \omega, M)$ are the longitude of the node, the argument or perigee and the mean anomaly; $\vartheta_{e}$ is the Greenwich sidereal time. Thus the time series of the geopotential along the orbit contains the frequencies

$$
\frac{d}{d t}\left(v_{l m p q}-\psi_{l m}\right)=(l-2 p+q) n-m \dot{\vartheta}_{e}+[(l-2 p) \dot{\omega}+m \dot{\Omega}]=\nu_{l m p q}
$$


where the dot stands for time derivative.

In the 2-body approximation, that is assuming that the mean motion $n$ is constant and the portion of the above formula between square brackets is zero, the geopotential is a finite sum of periodic terms with the frequencies

$$
\nu_{l m p q}=(l-2 p+q) n-m \dot{\vartheta}_{e} .
$$

The eccentricity functions $G_{l p q}(e)$ are of order $|q|$ in the eccentricity. Thus, for a nearly circular orbit such as the one of GOCE, the terms with $q \neq 0$ are less important. In a better approximation, the mean motion and the precession rates $\dot{\omega}$ and $\dot{\Omega}$ have to be computed taking into account the secular perturbations due to the $\bar{C}_{20}$ term; this changes the structure of the frequency spectrum for the $l=0$ zonal harmonics.

\subsection{Exact RESOnAnCe}

Let us suppose the orbit of the satellite is exactly resonant with the rotation of the Earth, that is there are two integers $h, k$ such that

$$
\frac{n+\dot{\omega}}{h}=\frac{\dot{\vartheta}_{e}-\dot{\Omega}}{k}=\mu .
$$

In the precessing ellipse approximation, this implies that the orbital period of the satellite is exactly $h / k$ sidereal days: the subsatellite track returns on the same curve with a repeat cycle of $h$ days. Neglecting the $q \neq 0$ frequencies, the frequencies appearing in the time series are then multiples of the basic frequency $\mu$

$$
\nu_{l m p 0}=[(l-2 p) h-m k] \mu .
$$

Thus the signal from all harmonics is periodic of period $2 \pi / \mu$ and can be described as a Fourier series with arguments $j \mu t$, with $j$ an integer.

The signal from the harmonics with the same $m$ and with an $l$ having the same parity share the same frequencies, with different amplitudes. In a frequencywise approach, after having computed the lumped coefficients represented by the amplitudes of the signal for each frequency, it is possible to solve for the individual geopotential coefficients, using a block diagonal structure with the harmonics with the same $m$ belonging to the same block. In this algorithm, the blocks contain significant offdiagonal terms (Sneeuw and van Gelderen, 1997, Figure 4.1). Even within a timewise approach, the normal matrix could be decomposed by the value of the order $m$, as proposed in (Colombo, 1989).

Since the individual Fourier terms $\cos (j \mu t)$ and $\sin (j \mu t)$ are orthogonal (the integral over one period of their product is zero), spherical harmonics generating signals with disjoint sets of frequencies are also 
orthogonal. In particular, two spherical harmonics with degree and order $(l, m)$ and $\left(l^{\prime}, m^{\prime}\right)$ are orthogonal in the time series if and only if

$$
k m^{\prime}= \pm k m(\bmod h) .
$$

A decomposition of the normal matrix by blocks corresponding to a remainder class is therefore exactly block diagonal, even taking into account the correlations between signals with different frequencies, neglected in a pure frequencywise approach. Because of the finite measurement time span, and especially of the data gaps, the observed signals with different frequencies are correlated, thus the off diagonal blocks with different $m$ in the same remainder class are not zero.

The above results refer to the geopotential, but in fact apply to whatever harmonic function expanded in spherical harmonics. If the geopotential is harmonic, then all its partial derivatives are also harmonic, including the gravity field and the gravity gradient. Moreover, the derivatives of a spherical harmonic with order $m$ has the same order $m$ (this is not true for the degree $l$ ).

Thus the first order perturbations on the spacecraft position (e.g., the GPS observables), being integrals of the time series of some gravity field component, follow the same orthogonality rule (Milani et al., 1998). The GOCE observables, being components of the gravity gradient, are also orthogonal in the time series unless the orders $m$ and $m^{\prime}$ fulfill Eq. (8).

Thus in the exact resonance case (and with negligible eccentricity) the coefficients in $g$ can be reordered by remainder class of the order $m$; that is, $C_{g g}^{r}$, the diagonal block number $r$, refers to the harmonic coefficients of degree and order $(l, m)$ such that $k m= \pm r(\bmod h)$. With this resonant decomposition the normal matrix $C_{g g}$ is exactly block diagonal. The advantage of this method with respect to the decomposition by order $m$ is that the number of blocks is smaller, allowing for a limited number of independent computations, and the correlations are not neglected.

\subsection{Approximate Resonance And Iterative Procedure}

For a realistic satellite orbit, a resonance condition such as Eq. (7) is not exactly fulfilled, and anyway the mean motion undergoes changes as the semimajor axis of the orbit changes (as a result of the drag and of the drag-controlling propulsion). Thus the problem is to find an approximate resonance, to assess the relative size of the off-diagonal blocks and to find an algorithm to take into account their contribution to the solution $\Delta g$ (and to the covariance matrix $\Gamma_{g g}$ ). 
As an example, for a semimajor axis of $6621.62 \mathrm{~km}$ the values of the mean frequencies (taking into account the secular perturbations due to the $\bar{C}_{20}$ term) are (in rad/s)

$$
n=1.1726 \times 10^{-3} \quad ; \quad \dot{\omega}=8.27 \times 10^{-7} \quad ; \quad \dot{\Omega}=-1.99 \times 10^{-7}
$$

thus the ratio

$$
\frac{n+\dot{\omega}}{\dot{\vartheta}_{e}-\dot{\Omega}}=16.05
$$

is close enough to $h / k=16 / 1$. However, the exact value of the semimajor axis for GOCE is not yet known, and it is expected that different phases of the mission will have a different mean semimajor axis (ESA, 1999, Section 6.2.4). Thus we have used the resonant decomposition corresponding to a resonance not too close to the nominal one, that is $h=31, k=2$, to test the robustness of the method.

An assessment of the relative size of the off-diagonal blocks was performed in the case of the GPS observables: the off-diagonal block correlations were found to be less than $1 \%$ in the most critical cases involving the $r=0$ block, less than $0.4 \%$ in the other cases (Milani et al., 1998, Figure 3.12). Although the gradiometer observables are somewhat different, they are not affected by the small divisor problems in the $r=0$ block, thus we expect similar results.

The simplified inversion of the $C_{g g}$ matrix by ignoring the off-diagonal blocks can be considered as a first approximation of an iterative procedure: let us rewrite the normal system of Eq. (5) as

$$
(M-N) \Delta g=D_{g}
$$

where $M$ is the block diagonal approximation, whose inverse $M^{-1}$ can be computed block by block. Then the standard iterative procedure to solve the linear system (9) is to transform to a fixed point problem (Bini, 1988, Section 5.2)

$$
M \Delta g=N \Delta g+D_{g} \Leftrightarrow \Delta g=M^{-1} N \Delta g+M^{-1} D_{g}=P \Delta g+Q
$$

which can be solved by iteration

$$
\Delta^{(1)} g=P \Delta^{(0)} g+Q ; \quad \Delta^{(2)} g=P \Delta^{(1)} g+Q \ldots
$$

starting from an arbitrary initial guess $\Delta^{(0)} g$; if $\|P\|<1$ the sequence $\Delta^{(k)} g$ converges to some $\Delta^{*} g$ fulfilling the fixed point equation and the complete normal system. If the initial guess is $\Delta^{(0)} g=\underline{0}$, then

$$
\Delta^{(1)} g=Q=M^{-1} D_{g}
$$

is the solution obtained with the approximate inversion. Thus the simplified computation can be justified as a first step of a convergent 


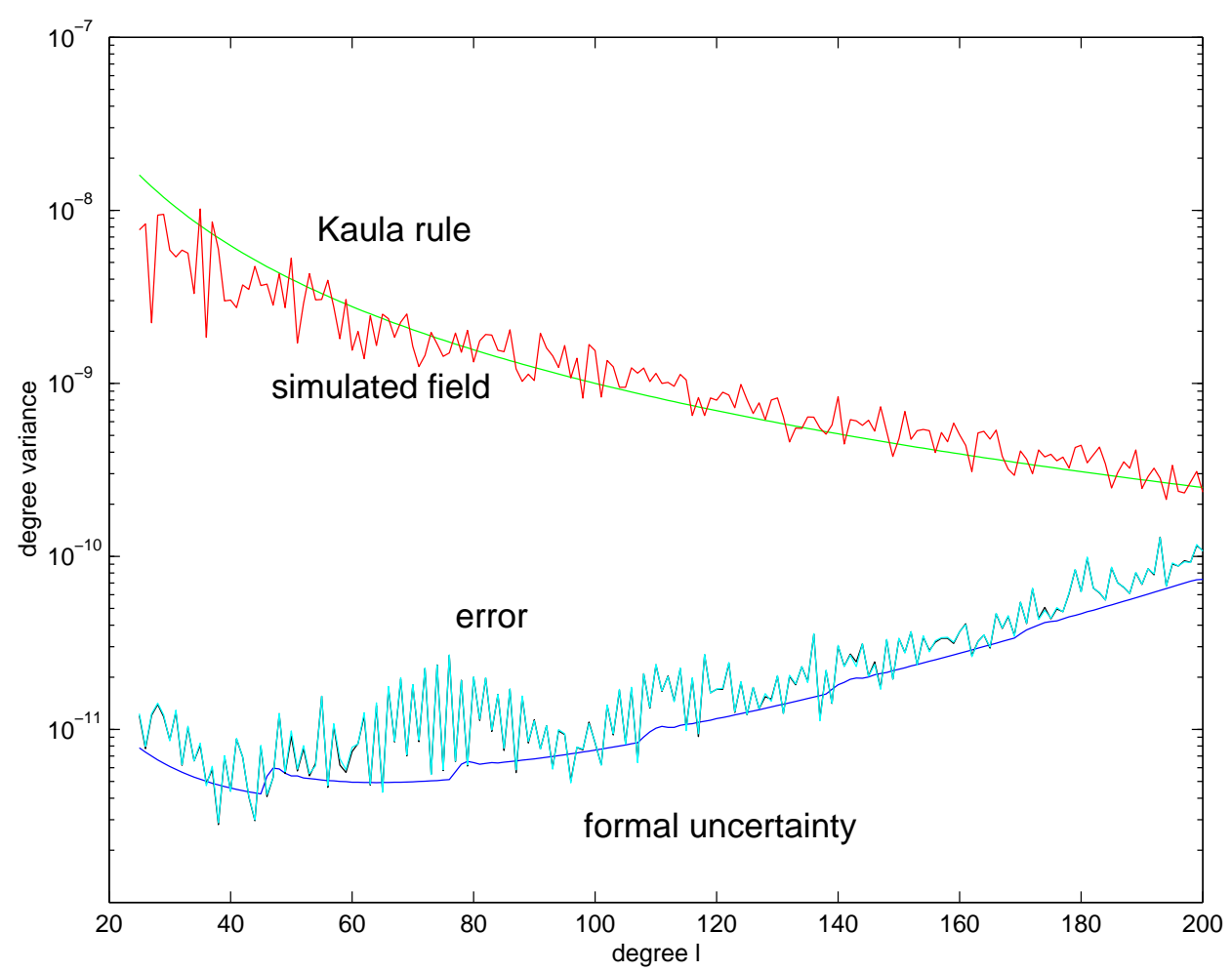

Figure 3. For the remainder class $r=1$, we plot as a function of the degree $l$, for $25 \leq l \leq 200$ : the gravity field signal (EGM96), the approximating Kaula's rule, the formal uncertainty and the actual error (estimate minus "true" value used in the data simulation) of the first and the second iteration. The last two curves are almost superimposed.

iteration. Figure 3 shows an example of the computation of the solution for the gravity coefficient, and their covariance, for the remainder class $r=1$, which includes the harmonics with $m=15,16, \ldots$.

The norm of $P$ is small because the off-diagonal correlations are small, but we do not have a quantitative estimate of this norm, thus we are unable to compute analytically how many iterations would be necessary for satisfactory convergence. To fill this gap in our knowledge, we perform a numerical test consisting of a computation of the second iteration $\Delta^{(2)} g$, which is possible by applying the correction of the first iteration $g \rightarrow g+\Delta^{(1)} g$ (for all remainder classes). This is possible only after a complete first iteration, 16 separate differential corrections with $r=0,1, \ldots, 15)$, by repeating the computation of the residuals and performing a second iteration for some remainder class.

Figure 3 shows both the result of the first and the second iteration, and the two curves are so close that most points are superimposed in the 
plot. We can conclude that, even by using $h / k=31 / 2$, not the most appropriate resonance for the value of $n$ of GOCE, the off-diagonal terms can be neglected and a full second iteration is not necessary ${ }^{6}$.

\section{Consequences of polar gaps}

In a solution for the geopotential coefficients up to a large degree and order $l_{\max }$, even after controlling the local-global correlations (with an appropriate choice of the minimum degree $l_{\text {min }}$, as discussed in Section 3.2), and after decomposing the normal matrix of the global parameters $C_{g g}$ as discussed in the previous Section, some diagonal blocks $C_{g g}^{r}$ are still very badly conditioned. As an example, the results for the remainder class $r=0$ in a solution for $l \leq l_{\max }=200$ are shown in Figure 4. The "belly" appearing both in the formal RMS and in the actual error is due to correlation among the zonal harmonics. The situation is such that the error to signal ratio reaches $\simeq 1$ around degree 100. The analogous figure for $l_{\max }=90$ would show a much lower belly, which does not result in an error to signal $\geq 1$.

It can be checked that the large RMS (and also actual errors) occur in this remainder class only for the zonals $m=0$; the other harmonics, with $m=31,62, \ldots$, are determined with error/signal ratio well below 1 for all $l$. The correlations are significant only among the zonals, e.g., the $l=150, m=0$ harmonic has correlations with all the other even zonals, $l=2-2 p, m=0$, with correlation 0.9999 with the $l=148$ and the $l=152$ one; there is also some correlation, although less extreme, with the odd $l$ zonals.

This is easy to understand by using the frequency analysis of Section 4.1: for $m=0$, low $e$ (thus the main terms have $q=0$ ):

$$
\nu_{l 0 p 0}=(l-2 p)(n+\dot{\omega})
$$

and the highly correlated harmonics share the same frequencies: consecutive even (or odd) zonals have only one frequency not in common.

Similar results are obtained for low $m$ : e.g., for $r=2$, that is for $m=1,29,33, \ldots$ the error to signal ratio reaches 1 around $l=90$. For $m \geq 7$ the formal and actual errors curves still show the "belly" at the intermediate degrees, but the error to signal ratio is below 1 . These difficulties in solving for the low $m$ coefficients with GOCE are known (Aguirre-Martinez and Sneeuw, 2003, Figure 4) and some explanation can be found in the literature (e.g., Sneeuw and van Gelderen, 1997).

\footnotetext{
${ }^{6}$ When processing the real data a second iteration may be necessary for other reasons, e.g., the correction to the gravity coefficients may be large.
} 


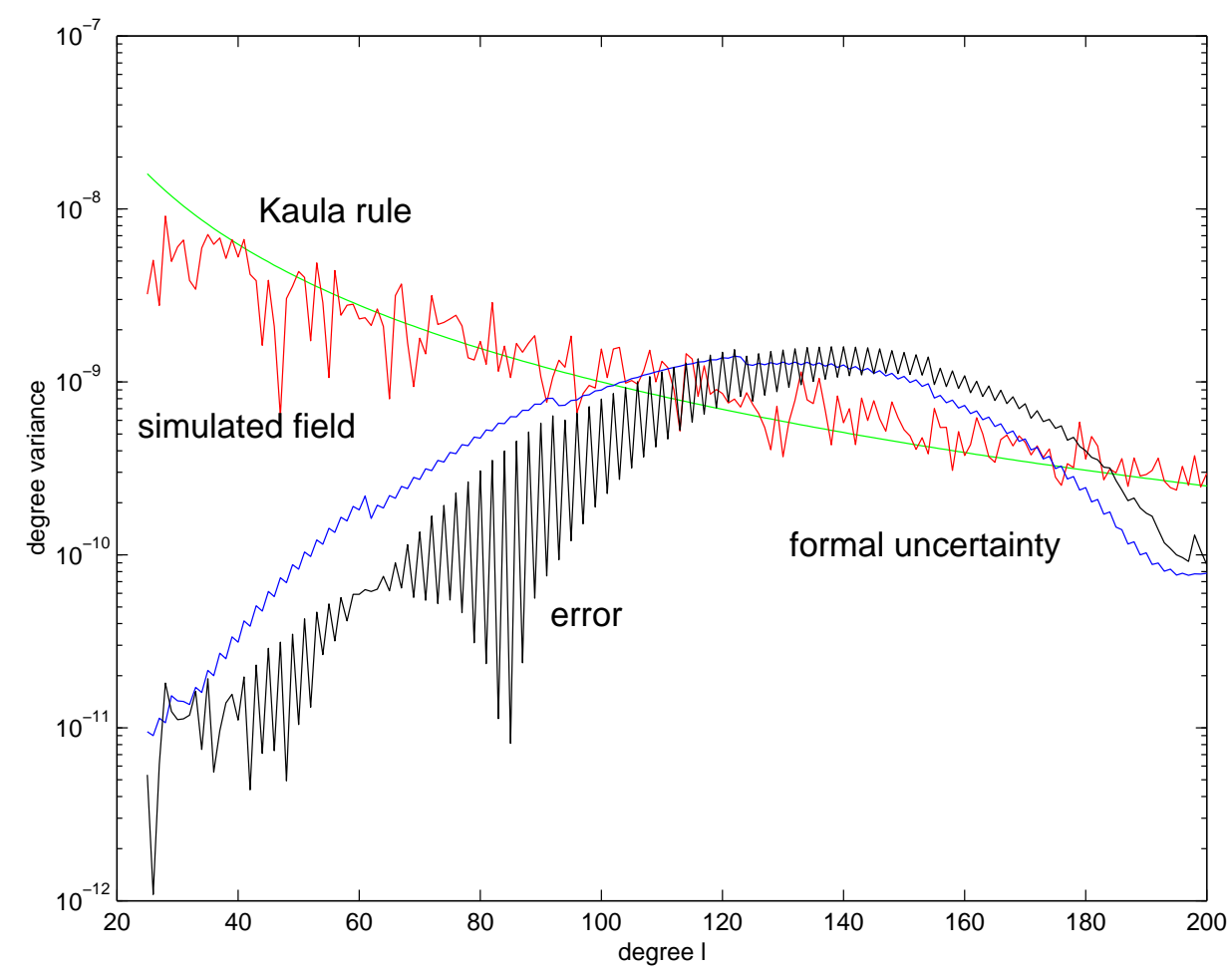

Figure 4. For the $r=0$ class, including the zonals, in a solution for $l \leq 200$, the formal and actual error are compared with the signal. The large bulge is such that both the formal and the actual error exceed the signal for degree $l>100$.

The problem is due to the polar gaps, that is to the fact that the nonpolar orbit of GOCE results in subsatellite tracks never crossing the two polar caps (with latitude above $\simeq 83^{\circ} .5$ and below $-83^{\circ} .5$ ). This is related to the non-orthogonality of the spherical harmonics over a latitude band (Albertella et al., 1999; Pail et al., 2001).

\subsection{Principal COMponents analysis}

Given the covariance matrix for the global parameters $\Gamma_{g g}$, or at least one of the blocks used in the resonant decomposition approximation $\Gamma_{g g}^{r}=\left[C_{g g}^{r}\right]^{-1}$, we can perform a principal components analysis. That is, let $\lambda_{1}>\lambda_{2}>\ldots>\lambda_{s}$ be the square roots of the eigenvalues of $\Gamma_{g g}^{r}$. It turns out that for the $r=0$ block the values are $2.2 \times 10^{-8}, 1.7 \times$ $10^{-8}, 3.3 \times 10^{-9}, 2.5 \times 10^{-9}, 7 \times 10^{-10}, \ldots$, that is the first two eigenvalues are similar and significantly larger than the following ones.

The corresponding unit eigenvectors $V_{j}, j=1, \ldots, s$ can be geometrically interpreted as the directions of the semiaxes of the confidence 


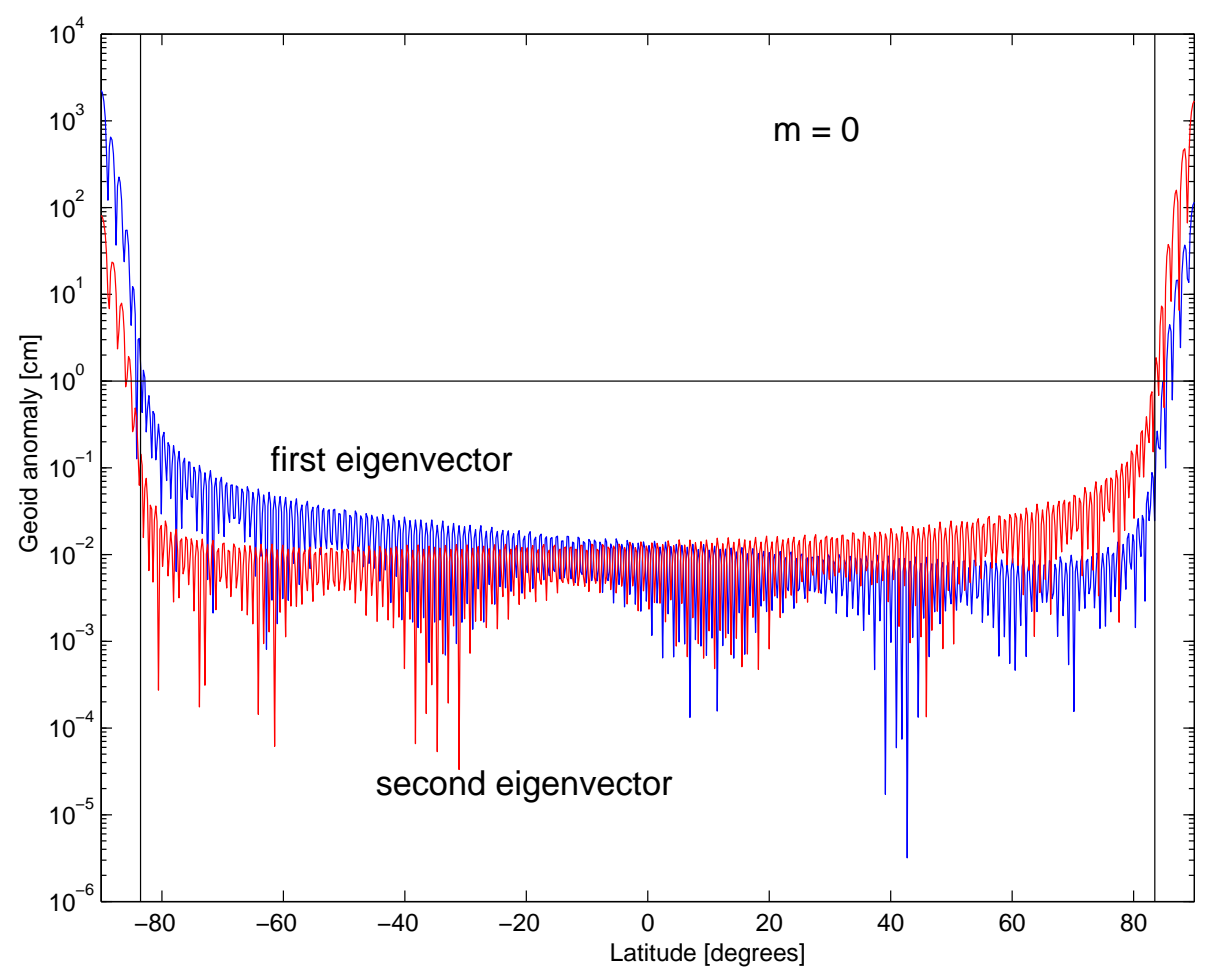

Figure 5. The gravity anomalies corresponding to the two principal axes of the confidence ellipsoid (for $\chi=1$ ) represented as geoid anomalies, as a function of latitude. The vertical lines bound the latitude band covered by GOCE, the horizontal line is at $1 \mathrm{~cm}$.

ellipsoid; the vectors $\lambda_{j} V_{j}$ are the semiaxes of the ellipsoid $Z(1)$. Each vector $\lambda_{j} V_{j}$ contains harmonic coefficients and represents an harmonic function. The components of the eigenvectors $V_{1}, V_{2}$ corresponding to harmonics with $m \neq 0$ are very small $\left(<10^{-4}\right)$, thus the harmonic functions with coefficients $\lambda_{1} V_{1}$ and $\lambda_{2} V_{2}$ are essentially zonal harmonics and they can be represented as a function of latitude. In Figure 5 we represent the two longest semiaxes of the confidence ellipsoid as geoid anomalies. The anomalies are concentrated on the two polar caps, with $\lambda_{1} V_{1}$ more pronounced on the $\mathrm{S}$ pole, $\lambda_{2} V_{2}$ on the $\mathrm{N}$ pole. The size of the anomalies is huge, up to $\simeq 20 \mathrm{~m}$ on the poles.

To understand why the GOCE results leave undetermined the geoid near the poles, we have represented in Figure 6 one of the GOCE observables, the radial component of the gravity gradient of the anomalies $\lambda_{1} V_{1}$ and $\lambda_{2} V_{2}$. Although the undetermined anomalies are not exclusively appearing on the polar caps, the signal for latitudes between $-83^{\circ} .5$ and $+83^{\circ} .5$ is well below the noise of the GOCE measurements. 


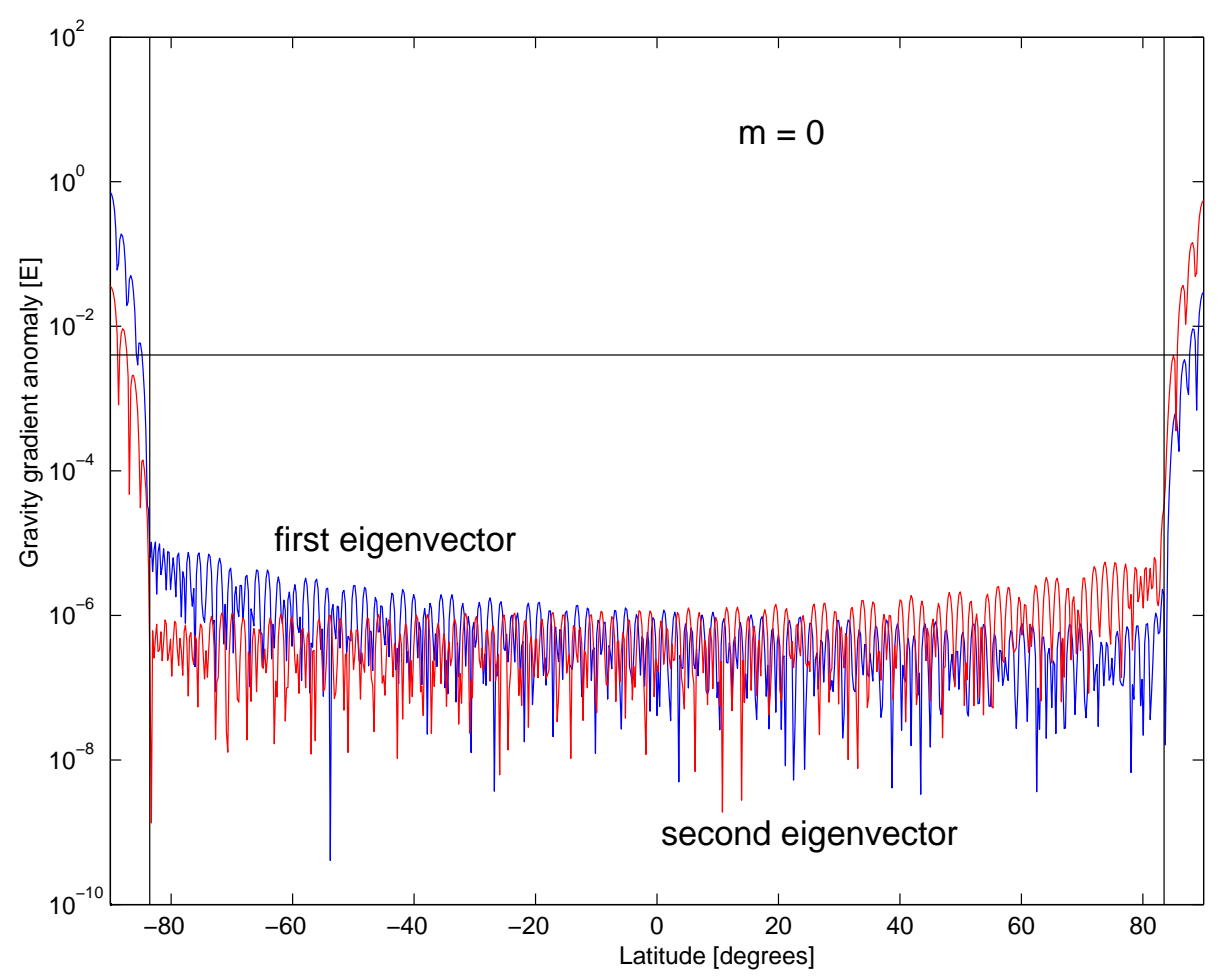

Figure 6. The gravity anomalies corresponding to the two principal axes of the confidence ellipsoid (for $\chi=1$ ) represented as gravity gradient anomalies, as a function of latitude. The horizontal line is at $0.004 \mathrm{E}$, the noise level for GOCE.

For the $r=2$ block the square roots of the eigenvalues are $2.95 \times$ $10^{-8}, 2.94 \times 10^{-8}, 2.22 \times 10^{-8}, 2.21 \times 10^{-8}, 3.2 \times 10^{-9}, \ldots$, that is the first four eigenvalues are of comparable value, two by two almost equal. Indeed, the harmonic functions defined by $\lambda_{2} V_{2}$ is obtained, with a good approximation, by a rotation of $90^{\circ}$ in longitude from $\lambda_{1} V_{1}$, and the same for $\lambda_{4} V_{4}$ from $\lambda_{3} V_{3}$. Thus in Figure 7 we have represented the first and the third principal axes. The value for a given longitude is the RMS of the anomaly over the corresponding parallel. Also in this case the values near the poles are large, $\simeq 10 \mathrm{~m}$.

\subsection{Symmetry and Degeneration}

What we would like to explain is why the undetermined anomalies are as large as shown in the Figures 5 and 7, and why the situation becomes worse as the solution is pushed to higher $l$.

Typically, the origin of an approximate degeneracy (that is, a poorly conditioned normal matrix) is due to a "nearby" complete degeneracy 


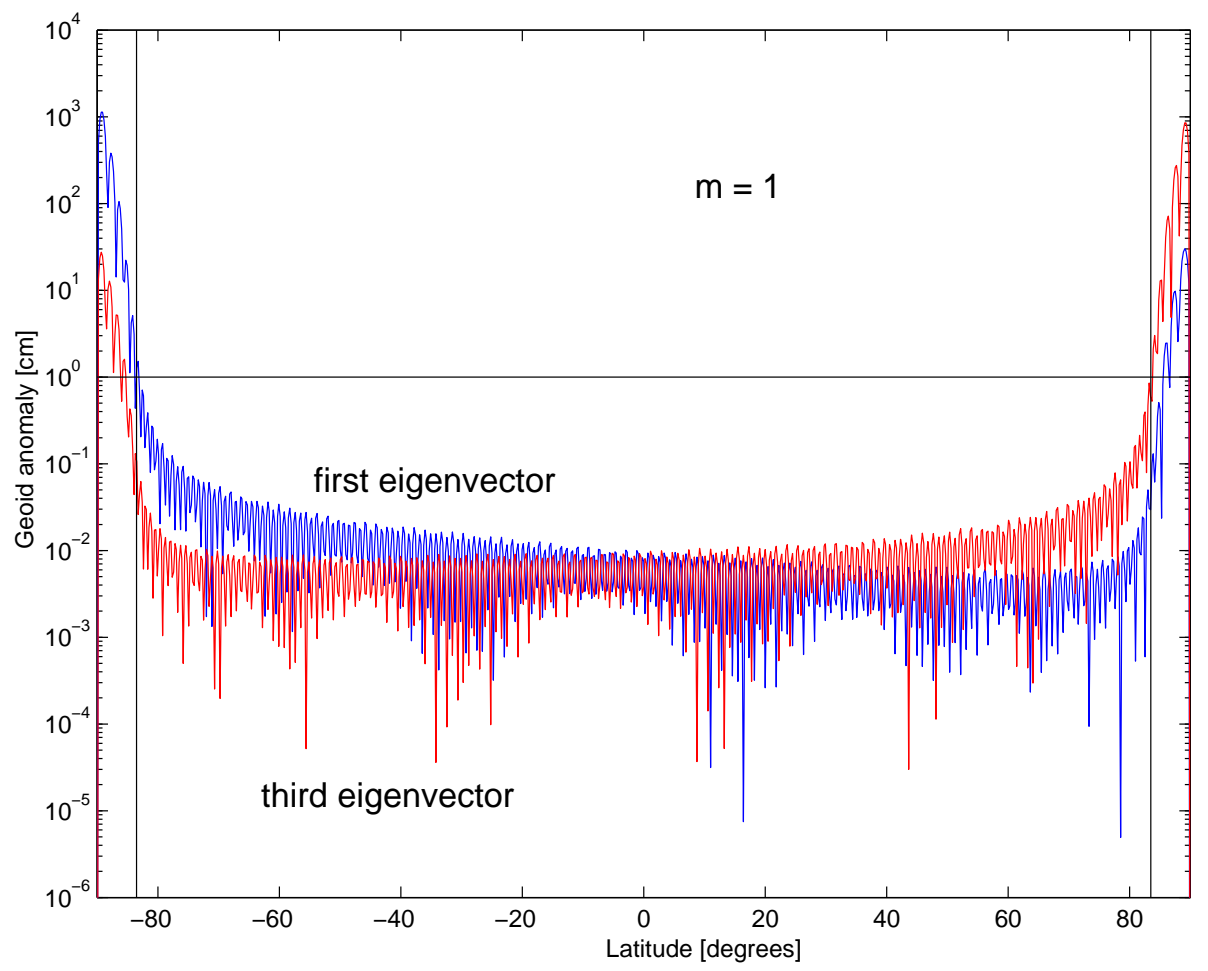

Figure 7. For the $r=2$ class, that is essentially for $m=1$, the geoid anomalies corresponding to the first and the third principal axes of the confidence ellipsoid. For each latitude we plot the RMS over the parallel. The curves for the second and the fourth principal axes would be the same.

(normal matrix with zero eigenvalues). That is, there must be an exact symmetry group $S$ of transformation in the parameter space $g$, such that $s(g)$, with $s \in S$, gives exactly the same observations as $g$, with the given measurement method and spatial/temporal distribution (Milani and Melchioni, 1989; Bonanno and Milani, 2002). The exact symmetry results in a complete degeneracy of the normal matrix, with the number of zero eigenvalues corresponding to the dimensions of the symmetry group. When this symmetry is broken it remains as an approximate symmetry, and the zero eigenvalues are replaced by very small ones.

The case of GOCE is somewhat more complicated, because the symmetry group is an infinite dimensional space of harmonic functions. Its existence can be proven by selecting, on the sphere of radius $R_{\oplus}+h$ ( $h$ being the mean altitude of the satellite), an arbitrary function $\Phi$ with support in the polar caps (that is, the function is exactly zero for latitudes between $-83^{\circ} .5$ and $83^{\circ} .5$ ). By the Dirichlet theorem (Heiskanen and Moritz, 1967, Section 1-16) there is a function harmonic for 
geocenter distance $\geq R_{\oplus}+h$ and coinciding with $\Phi$ on the sphere of radius $R_{\oplus}+h$. Note that such function may not exist on the sphere of radius $R_{\oplus}$ because the downward continuation may well be divergent: that is, it does not need to be a "realistic" gravity anomaly.

If we take the approximation that GOCE flies at an exactly constant altitude, then the gradiometer is measuring the second derivatives where $\Phi$ is zero. Thus the derivatives along the horizontal directions are zero, and because $\Delta \Phi=0$, the radial gravity gradient is zero too.

This exact symmetry is broken for two reasons: first, GOCE does not fly exactly at a constant altitude, although the eccentricity is small $(e<0.0045)$; moreover, the inclination is not exactly constant, thus the avoided caps are not exactly bounded by a parallel. Second, the harmonic function we are trying to fit to the GOCE data is the sum of only a finite number of harmonics, with limited degree $l \leq l_{\max }$.

The cap function $\Phi$ cannot have a finite spherical harmonics expansion, because it is not an analytic function on the sphere. If a cap function is expanded in a finite sum of spherical harmonics, that is its representation as spherical harmonics series is truncated to degree $l_{\text {max }}$, the remainder of the series has a small RMS $\sigma\left(l_{\max }\right)$ on the latitude band where $\Phi$ is zero, with $\sigma\left(l_{\max }\right) \rightarrow 0$ for $l_{\max } \rightarrow+\infty$. This is what is shown in the Figures 5 and 7 , and the corresponding observable signal for GOCE is not zero but very small, as in Figure 6.

Thus it is obvious that the "belly" must become more and more pronounced as $l_{\max }$ increases; at the limit for $l_{\max } \rightarrow+\infty$ the geoid anomaly on the sphere of radius $R_{\oplus}$ may well diverge.

\section{Error sources}

The results for all the remainder classes, that is for all the gravity harmonics of degrees $25 \leq l \leq 200$, are summarized in Figure 8 . The formal error is of course larger than the one of the naive estimate of Figure 2, but only because of the polar gaps. Indeed, for the classes not including low values of $m$ the figures look like the one for $r=1$ (Figure 3). That is, our formal error is as good as it can be, taking properly into account the polar gaps.

It would be possible to control the "belly" either by using Kaula's rule collocation, or by constraining the behavior on the unobserved polar caps (Sneeuw and van Gelderen, 1997). However, this would give illusory formal errors for the individual coefficients, and anyway the values of the anomalies on the polar caps would be an artefact.

The actual error follows the trend of the formal error as a function of $l$, but it is somewhat higher, by a factor $\simeq 2$. This should include 


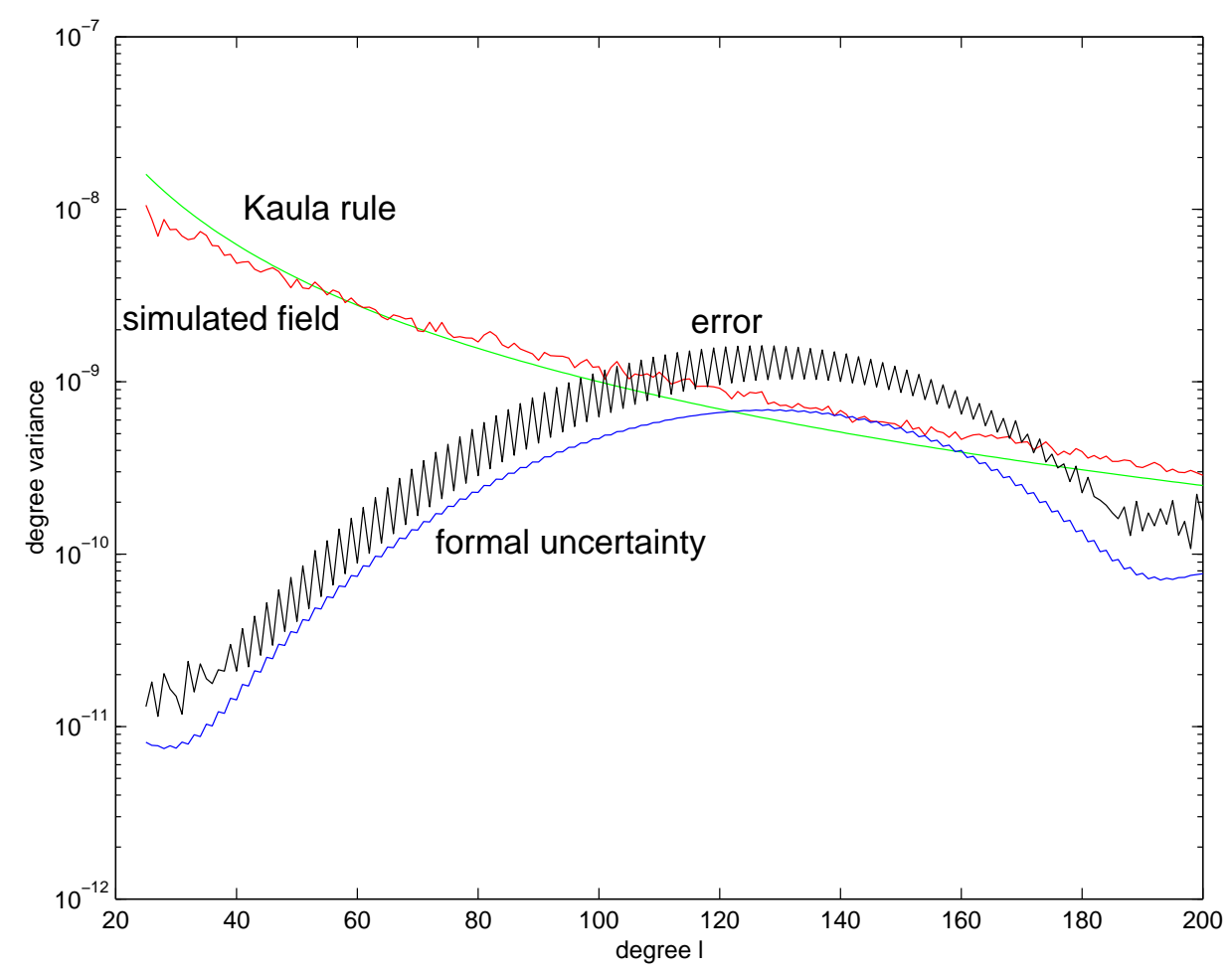

Figure 8. For all the remainder classes together, in a solution for $l \leq 200$, the formal and actual error are compared with the signal.

the effect of all the systematic error sources, including the systematic components of our measurement error model, the orbital error and the spurious signal either unfiltered or introduced by our calibration fit. In this Section we try to establish which of these is dominant, that is what is the fundamental limitation of the GOCE experiment.

\subsection{Gradiometer Calibration}

To assess the origin of the actual errors exceeding the formal ones, it is important to analyze the specific harmonics where the largest actual/formal error ratio takes place. As an example, for the remainder class $r=7$ the actual error grows significantly for $l>160$, to the point of reaching an error/signal ratio of 1 (see Figure 9). Among the harmonics in this remainder class, the ones with $m=167$ show a particularly high actual/formal error ratio.

We have investigated the possibility that the "boundary jumps" introduced by our fit to the calibration model were responsible. Figure 9 


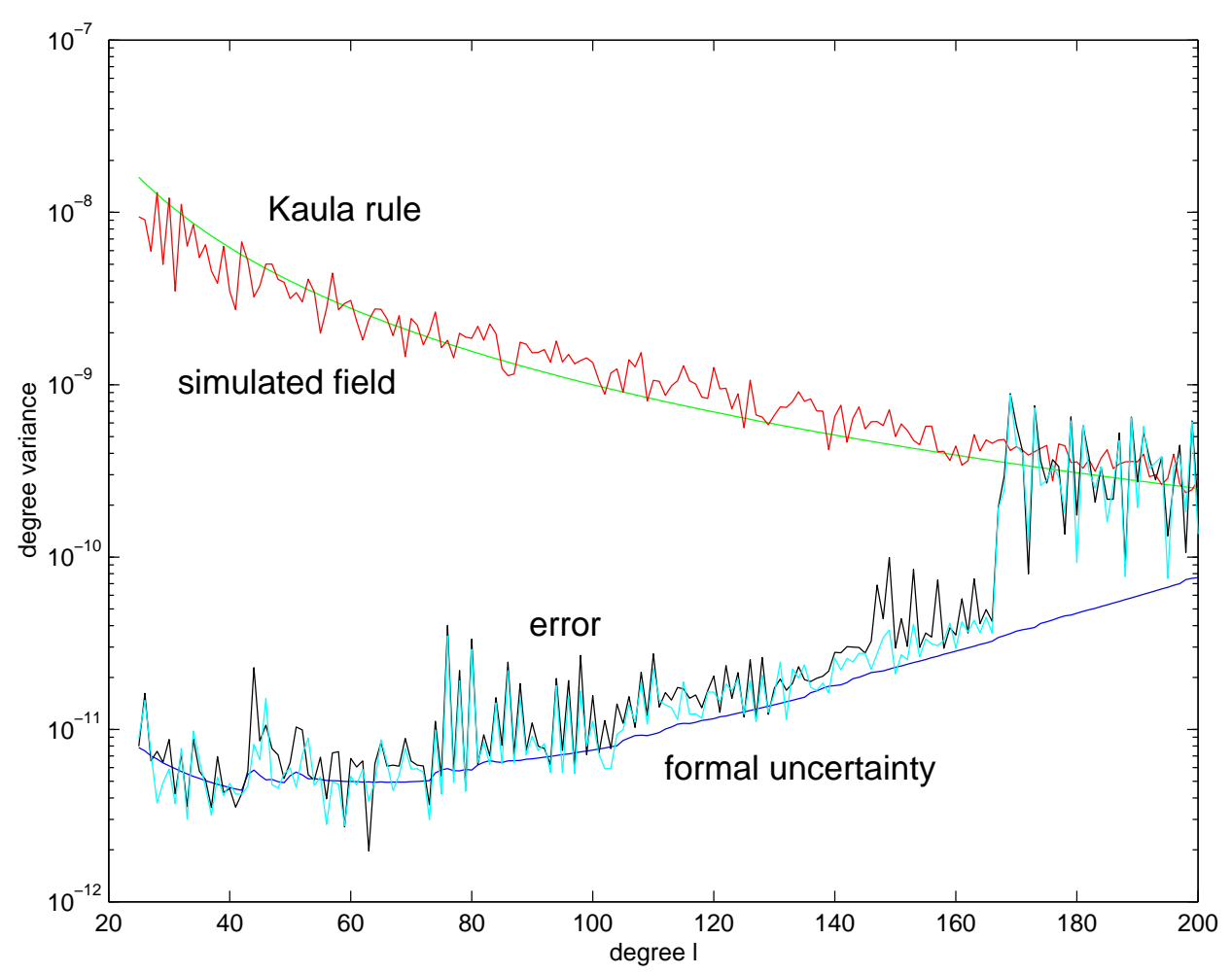

Figure 9. For the $r=7$ remainder class the formal and actual error are compared with the signal in the case with and without extended arc boundaries. The two curves of the actual errors are almost superimposed.

also shows the $r=7$ solution with margins beyond the arc boundary; the effect on the actual error is negligible.

Thus the only interpretation is that the spurious signal with frequency $1 / 1000 \mathrm{~Hz}$ we have introduced in the gradiometer biases is responsible. Such signal, as discussed in Sec. 2.3, is not removed by the calibration fit because it has a frequency above the calibration band limit $f_{c}=1 / 2000 \mathrm{~Hz}$. Looking for gravity field signals with similar frequencies, we find, e.g., that for $l-2 p=-5, m=167$ the frequency $\nu_{l m p 0}$ corresponds to a period of $990 \mathrm{~s}$; for $l-2 p=-19, m=167$ the frequency is negative, with a period of $957 \mathrm{~s}$. It can be argued that the spurious signal results in an aliasing between the gradiometer bias and the gradiometer signal for some harmonics with nearby frequencies. The value $1000 \mathrm{~s}$ for the period in our systematic error model is arbitrary, but any other period would generate aliasing with some harmonics. 


\subsection{ORBIT ERROR}

Because of the separation of the POD from the fit for the $\ell$ and $g$ parameters, we are not including an orbit error model in our covariance matrices. Thus we have to ask if the orbit error which has to be expected can result in significant systematic errors in the gravity coefficients.

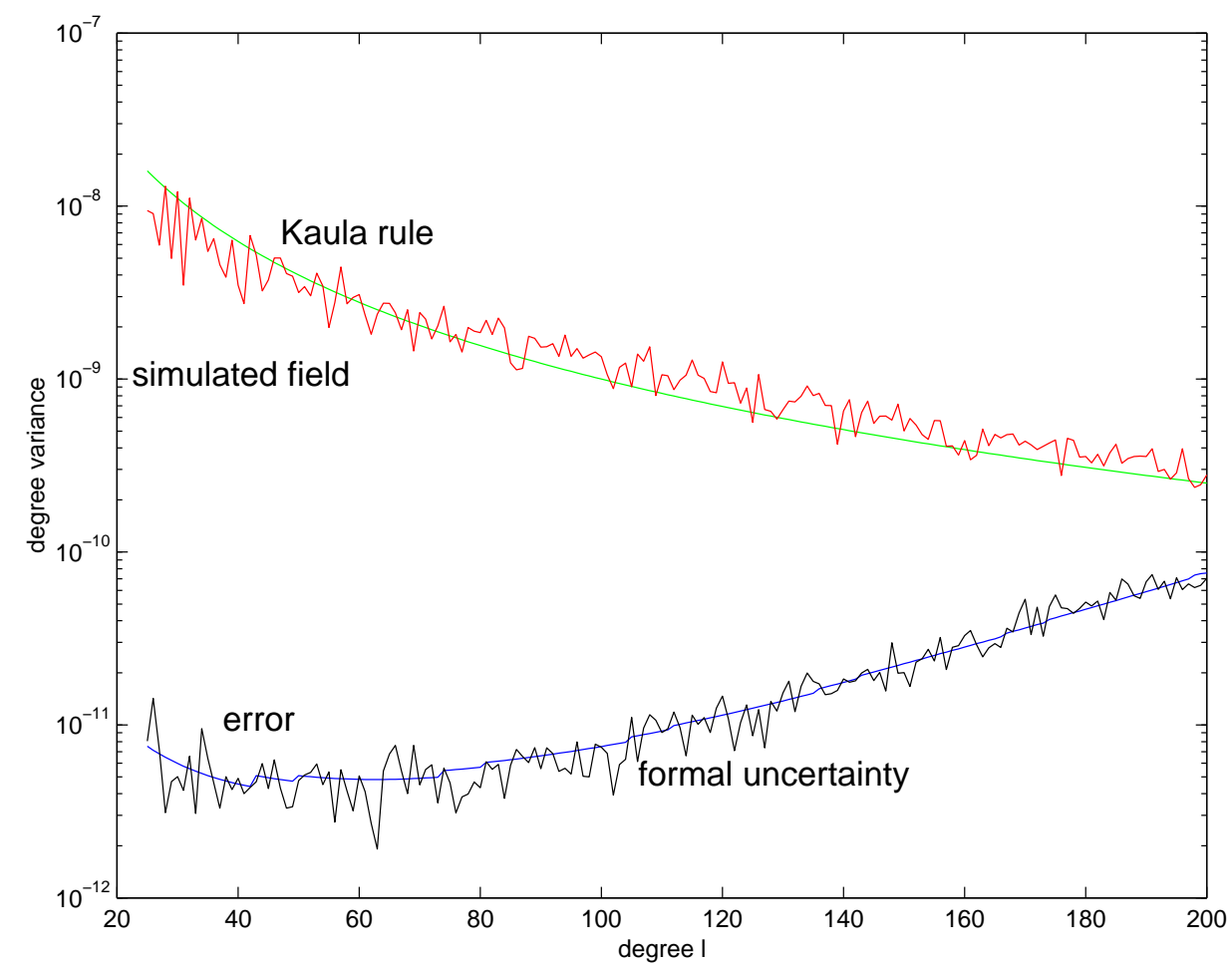

Figure 10. For the $r=7$ remainder class the formal and actual error are compared with the signal in the case where only the orbit error is included but no gradiometer bias. To be compared with Figure 9 .

In our data simulations we have always included an error in the satellite position coordinates with a random component (Gaussian uncorrelated, $\mathrm{RMS}=2 \mathrm{~cm}$ ) and systematic components with periods one year, one day, once per rev and twice per rev, all with amplitude $3 \mathrm{~cm}$. To test the relevance of this orbit error on the solution for the gravity coefficients we have performed a simulation without the gradiometer bias, with the orbit errors as the only systematic error source: as shown in Figure 10, the actual error in the coefficients becomes statistically consistent with the formal error. Thus the actual errors in excess with respect to the formal ones (shown in Figure 9) have to be attributed 
to the effect of the systematic calibration bias not removed by our calibration procedure, while the orbit error does not matter.

\subsection{Expansion to higher DEGREes}

Another potentially important source of error in the solution for the gravity coefficient is the omission error. Whatever we choose for the maximum degree $l_{\max }$ of the harmonics included in the simulation, the harmonics with $l>l_{\max }$ will appear in the gradiometer signal and will not be solved as such, but may alias with the signal from lower harmonics, leading to systematic errors.

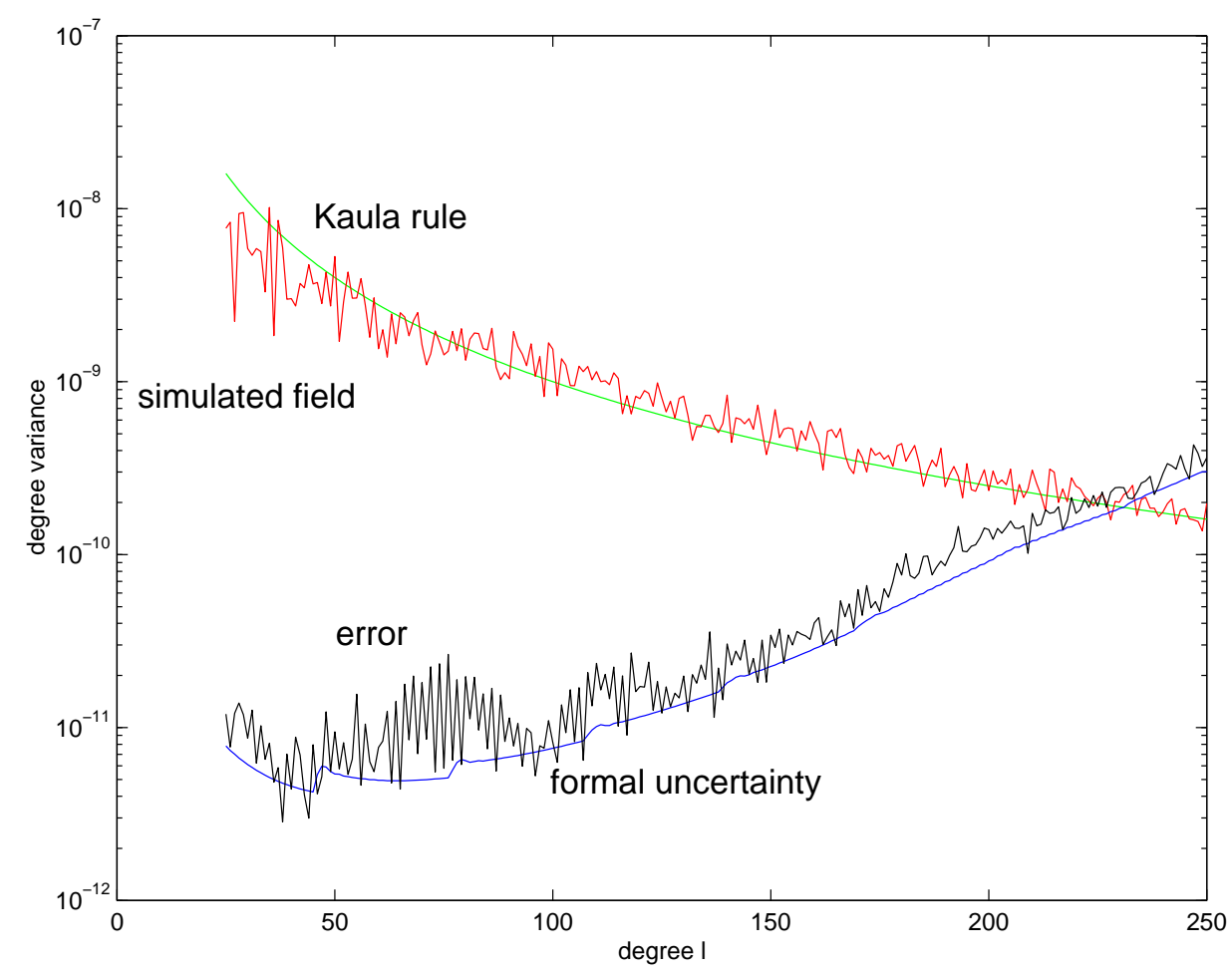

Figure 11. For the $r=1$ remainder class, in a solution for $l \leq 250$, the formal and actual error become larger than the signal for large $l$.

This effect can be tested by performing a data simulation with a gravity field extending to degree $>l_{\max }$, then solving only up to $l_{\max }$. A test was done with the data generated by the EGM96 field up to $l=250$ and the solution with $l_{\max }=200$. The results clearly showed that the effect on the actual error is not significant. Thus it is not necessary to take into account the influence of the higher degree coefficients by some formalism such as consider covariance. 
Another related question is the following. For the remainder classes not affected by the polar gap the error to signal ratio is still $\simeq 1 / 3$ at degree $l=200$ : would it be possible to extend the solution to higher degrees? The results of a solution for $25 \leq l \leq 250$ are plotted in Figure 11, showing that it is possible to solve for the harmonics of degree up to $l=220$ with error to signal ratio $\leq 1$. Note that even the formal error becomes larger than the signal for $l>230$, thus there is a limitation to the maximum degree of a significant solution even in the "perfect experiment" assumption of no systematic errors. As a general rule, the formal covariance result is a lower bound for the uncertainty, the actual results can only be worse.

\section{Conclusions}

We have defined an algorithm for the processing of the GOCE data, based on a multistage decomposition of the problem. As a result we have been able to perform a full simulation, including data simulation and differential corrections, with PC class computer resources. We have improved the theory explaining the remaining uncertainties in the solution for the gravity coefficients, resulting from the polar gaps.

We have reached the following conclusions. The harmonic coefficients can be solved for degree $l$ ranging between $\simeq 25$ and $200 \div 220$, with larger uncertainties for the low order $(m)$ harmonics, due to the polar gaps. These uncertainties for low $m$ do not affect the capability to solve accurately for the geoid outside of the polar caps.

We have found that the fundamental limitation of GOCE is in the difficulty of calibrating the gradiometer at the required level of accuracy. This is not a surprise: the calibration of accelerometers is one of the main problems of the new generation satellite geodesy missions (Bruinsma et al., 2003). The interesting result is that we have reduced the impact of the calibration problems to a factor $\simeq 2$ in the ratio actual/formal error. Even our result, however, uses some hypothesis on the behavior of the gradiometer errors not only in the measurement band, but also in a "safety band" between the calibration band (frequencies below $f_{c}=1 / 2000 \mathrm{~Hz}$ ) and the measurement band (with frequencies above $f_{m}=1 / 200 \mathrm{~Hz}$ ). We have assumed that there can be spurious signals in this band, but with amplitude growing only with the inverse of the frequency. Still these spurious signals are responsible for most of the increase of the actual with respect to the formal error.

Of course the choice of $f_{c}$ is somewhat arbitrary, and indeed we could have increased this frequency, e.g., by a factor 2 by increasing the total number of local calibration parameters to be solved, either 
by decreasing $\Delta t$ or by increasing $K$ in Eq. (2). The point is not to remove a spurious signal of known shape we have introduced in the data simulation according to a very simple model, but what should be done with the real signal, when the information on the shape of the signal will not be available. In any case some safety band is required, and the real quality of the result (as opposed to the formal one) will depend upon the level of control on the spurious, systematic signals in that band. As an example, the increase of the systematic effects from the attitude control resulting from giving up the electric propulsion attitude actuators (Catastini et al., 2004) especially affects the safety band. How to control the situation of the spurious signals in the frequency domain immediately below $f_{m}$ is going to be the main challenge of the GOCE operations and data processing.

Acknowledgments The theory of the resonant decomposition was originally developed, in collaboration with D. Vokrouhlický, in the framework of the SAGE project. We acknowledge useful discussions with F. Sansò and his team, in particular on the comparison and interaction between timewise and spacewise methods. This work was funded by Agenzia Spaziale Italiana (ASI) under contract I/R/194/02.

\section{References}

Aguirre-Martinez, M. and Sneeuw, N.: 2003, 'Needs and tools for future gravity measuring missions', Space Science Reviews, 108, 409-416.

Albertella, A., Sansò, F. and Sneeuw, N.: 1999 ,'Band-limited functions on a bounded spherical domain: the Slepian problem on the sphere', J. Geod. 73, 436-447.

Albertella, A., Migliaccio, F., Reguzzoni, M. and Sansò, F.: 2001, 'Spacewise approach and measurement bandwidth in satellite gradiometry', In: Proceedings of IAG 2001 Scientific Assembly, Budapest, 2-7.

Bini, D., Capovani, M. and Menchi, O. : 1988, Metodi numerici per l'algebra lineare, Zanichelli, Bologna.

Bonanno, C and Milani, A. : 2002, 'Symmetries and rank deficiency in the orbit determination around another planet', CMDA 83, 17-33.

Bouman, J. and Radboud, K.: 2003, 'Geodetic methods for calibration of Grace and GOCE', Space Science Reviews 108, 293-303.

Bruinsma, S., Loyer, S., Lemoine, J.-M., Perosanz, F. and Tamagnan, D.: 2003, 'The impact of accelerometry on CHAMP orbit determination', J. Geod. 77, 86-93.

Catastini, G., Andreis, D., Cesare, S., De Sanctis, S., Dumontel, M., Floberghagen, R., Lamarre, D., Parisch, M., Saponara, M. and Sechi, G.: 2004, 'The GOCE Endto-End System Simulator', In Proceedings of the "Second International GOCE User Workshop", Frascati March 8-10, in press.

Colombo, O.L.: 1989, 'Advanced techniques for high-resolution mapping of the gravitational field', in: Sansò, F. and Rummel, R.(eds.), Theory of Satellite Geodesy and Gravity Field Determination, Lecture Notes in Earth Sciences, Vol. 25, Springer-Verlag, 335-369. 
European Space Agency (1999) Gravity field and steady-state ocean circulation Mission, ESA-SP 1233(1), European Space Agency, Noordwijk

Heiskanen, W.A. and Moritz, H.: 1967, Physical Geodesy, Freeman \& Co, San Francisco.

Kaula, W.M.,1966, Theory of satellite geodesy, Blaisdell, Waltham, Massachusetts.

Lemoine, F.G., Kenyon, S.C., Factor, J.K., Trimmer, R.G., Pavlis, N.K., Chinn, D.S., Cox, C.M., Klosko, S.M., Luthcke, S.B., Torrence, M.H., Wang, Y.M., Williamson, R.G., Pavlis, E.C., Rapp, R.H. and Olson, T.R.: 1998, The Development of the Joint NASA GSFC and NIMA Geopotential Model EGM96, NASA/TP-1998-206861, NASA Goddard Space Flight Center, Greenbelt, Maryland, USA.

Milani, A. and Melchioni, E.: 1989, 'Determination of a local geodetic network by multi-arc processing of satellite laser ranges', in: Sansò, F. and Rummel, R.(eds.), Theory of Satellite Geodesy and Gravity Field Determination, Lecture Notes in Earth Sciences, Vol. 25, Springer-Verlag, 417-445.

Milani, A., Carpino, M., Rossi, A., Catastini, G. and Usai, S.: 1995, 'Local Geodesy by Satellite Laser Ranging: an European Solution', Manuscripta Geodetica 20, $123-138$.

Milani, A., Rossi, A. and Vokrouhlický, D.: 1998, 'The timewise approach', in: Albertella, A. and Migliaccio, F. (eds), SAGE, Satellite Accelerometry for Gravity Field Exploration: Phase A Final Report, International Geoid Service, Milano.

Milani, A., Rossi, A., Vokrouhlický, D., Villani, D. and Bonanno, C.: 2001, 'Gravity field and rotation state of Mercury from the BepiColombo Radio Science Experiments', Planetary and Space Science, 49, 1579-1596.

Pail, R., Plank, G. and Schuh, W.D.:2001, 'Spatially restricted data distributions on the sphere: the method of orthonormalized functions and applications', J. Geod. $\mathbf{7 5}, 44-56$.

Pail, R. and Plank, G.: 2002, 'Assessment of three numerical solution strategies for gravity field recovery from GOCE satellite gravity gradiometry implemented on a parallel platform', J. Geod. 76, 462-474.

Reigber, C.: 1989, 'Gravity Field Recovery from Satellite Tracking Data', in: Sansò F. and Rummel, R. (eds.), Theory of Satellite Geodesy and Gravity Field Determination, Lecture Notes in Earth Sciences, Vol. 25, Springer-Verlag, 197-234.

Reigber, C., Balmino, G., Schwintzer, P., Biancale, R., Bode, A., Lemoine, J.-M., Koenig, R., Loyer, S., Neumayer, H., Marty, J.-C., Barthelmes, F., Perosanz, F. and Zhu, S. Y.: 2002, 'A high quality global gravity field model from CHAMP GPS tracking data and Accelerometry (EIGEN-1S)', Geophys. Res. Lett., 29(14), $37-41$.

Rummel, R., van Gelderen, M., Koop, R., Schrama, E., Sansò, F., Brovelli, M., Migliaccio, F., Sacerdote, F.: 1993, 'Spherical harmonic analysis of satellite gradiometry', Publications on Geodesy, New Series 39, Netherlands Geodetic Commission.

Sneeuw, N. and van Gelderen, M.: 1997, 'The polar gap'. In: F. Sansò, R. Rummel, (eds.); Geodetic Boundary Value Problems in View of the One Centimeter Geoid, Lecture Notes in Earth Sciences, Springer Verlag, Berlin, 559-568.

Visser, P.N.A.M. and van den IJssel, J.:2000, 'GPS-based precise orbit determination of the very low Earth-orbiting gravity mission GOCE', J. Geod., 74, 590-602. 